\title{
Using synthetic MR images for field map-less distortion correction
}

David F Montez ${ }^{\dagger, \mathrm{a}, \mathrm{b}, *}$, Andrew N Van ${ }^{\dagger, \mathrm{a}, \mathrm{c}}$, Ryland L Miller ${ }^{\mathrm{a}, \mathrm{b}}$, Nicole A Seider ${ }^{\mathrm{a}}$, Scott Marek $^{\mathrm{b}}$, Annie Zheng ${ }^{\mathrm{a}}$, Dillan J Newbold ${ }^{\mathrm{a}}$, Kristen Scheidter ${ }^{\mathrm{a}, \mathrm{b}}$, Eric Feczko ${ }^{\mathrm{d}, \mathrm{e}}$, Anders J Perrone $^{\mathrm{d}, \mathrm{f}}$, Oscar Miranda-Dominguez ${ }^{\mathrm{d}, \mathrm{e}}$, Eric A Earl ${ }^{\mathrm{d}, \mathrm{f}}$, Benjamin P Kay ${ }^{\mathrm{a}}$, Abhinav K $\mathrm{Jha}^{\mathrm{c}, \mathrm{g}}$, Aristeidis Sotiras ${ }^{\mathrm{g}, \mathrm{h}}$, Timothy O Laumann ${ }^{\mathrm{b}}$, Deanna J Greene ${ }^{\mathrm{i}}$, Evan M Gordon ${ }^{\mathrm{g}}, \mathrm{M}$ Dylan Tisdall $^{\mathrm{j}}$, Andre Van Der Kouwe ${ }^{\mathrm{k}, \mathrm{l}}$, Damien A Fair ${ }^{\mathrm{d}, \mathrm{e}, \mathrm{m}}$, Nico U F Dosenbach ${ }^{\mathrm{a}, \mathrm{c}, \mathrm{g}, \mathrm{n}}$

${ }^{a}$ Department of Neurology, Washington University School of Medicine, MO, 63110, St. Louis

${ }^{b}$ Department of Psychiatry, Washington University School of Medicine, MO, 63110, St. Louis

${ }^{c}$ Department of Biomedical Engineering, Washington University School of Medicine, MO, 63110, St. Louis

${ }^{d}$ Masonic Institute for the Developing Brain, University of Minnesota Medical School, MN, 55455, Minneapolis

${ }^{e}$ Department of Pediatrics, University of Minnesota Medical School, Minneapolis, 55455, MN

${ }^{f}$ Department of Psychiatry, Oregon Health and Science University, OR, 97239, Portland

${ }^{g}$ Department of Radiology, Washington University School of Medicine, MO, 63110, St. Louis

${ }^{h}$ Institute for Informatics, Washington University School of Medicine, MO, 63110, St. Louis

${ }^{i}$ Department of Cognitive Science, University of California, San Diego, La Jolla, 92093, CA

${ }^{j}$ Department of Radiology, Perelman School of Medicine, University of Pennsylvania, Philadelphia, 19104, $P A$

${ }^{k}$ Department of Radiology, Harvard Medical School, Boston, 02115, MA

${ }^{l}$ Department of Radiology, Massachusetts General Hospital, Athinoula A. Martinos Center for Biomedical Imaging, Charlestown, 02129, MA

${ }^{m}$ Institute of Child Development, University of Minnesota Medical School, Minneapolis, 55455, MN

${ }^{n}$ Department of Pediatrics, Washington University School of Medicine, St. Louis, 63110, MO

\begin{abstract}
Functional MRI (fMRI) data acquired using echo-planar imaging (EPI) is highly distorted by magnetic field inhomogeneity. Distortion combined with underlying differences in image contrast between EPI and T1-weighted and T2-weighted (T1w/T2w) structural images makes the alignment of functional and anatomical images a challenge. Typically, separately acquired field map data are used to correct fMRI distortions and a flexible cost function insensitive to cross-modal differences in image contrast and intensity is used for aligning fMRI and anatomical images. The quality of alignment achieved with this approach can vary greatly and depends on the availability and quality of field map data. To address this issue, we developed Synth, a software package for distortion correction and cross-modal image registration that does not require separately acquired field map data. Synth combines information from $\mathrm{T} 1 \mathrm{w} / \mathrm{T} 2 \mathrm{w}$ anatomical images to construct an idealized undistorted synthetic image that has similar contrast and intensity properties to fMRI data. The undistorted synthetic image then serves as an effective reference for individual-specific nonlinear unwarping to correct fMRI distortions. We demonstrate that Synth reliably outperforms other standard registration and distortion correction approaches that utilize field maps in both pediatric (ABCD: Adolescent Brain Cognitive Development) and adult (MSC: Mid-
\end{abstract}


night Scan Club) data. Field map-less distortion correction with Synth allows accurate and precise registration of fMRI data with missing or corrupted field map information.

\section{Introduction}

BOLD-weighted (blood-oxygenation level dependent) functional MRI (fMRI) data consisting of echo planar imaging (EPI) images are severely distorted by inhomogeneities affecting the primary magnetic field [1-4]. EPI distortion, which consists of localized spatial deformation and loss of BOLD signal intensity, prominently affects areas of an EPI image containing large local differences in magnetic susceptibility. Due to the apposition of diamagnetic tissue and paramagnetic air in the sinuses and ear canals, areas of an EPI image that contain orbitofrontal cortex or the inferior temporal lobes often suffer from the most severe distortion. The susceptibility-induced artifacts are spatially non-uniform and therefore interfere with the performance of registration algorithms used during fMRI preprocessing to bring BOLD-weighted EPI images (predominantly $\mathrm{T}^{*}$-weighted images where $\mathrm{TE}$ is approximately equal to gray matter $\mathrm{T} 2 *$ ) into alignment with their associated anatomical images $(\mathrm{T} 1 \mathrm{w} / \mathrm{T} 2 \mathrm{w})$.

Understanding the relationship between brain anatomy and function is a key goal of systems neuroscience. Regardless of study design or choice of analysis space, registration and removal of EPI distortion to improve the correspondence between BOLD-weighted functional images and $\mathrm{T} 1 \mathrm{w} / \mathrm{T} 2 \mathrm{w}$ anatomical images is a crucial step in the analysis of fMRI data. For example, poor alignment counteracts the benefits of analyses performed on subject-specific regions of interest, defined with reference to a subject's anatomical images. Improper alignment of BOLD-weighted and anatomical images also degrades the performance of procedures that project volumetric fMRI data onto mesh surfaces derived from tissue segmentation [5]. The effects of poor registration and distortion correction also carry forward into group analyses. In group studies, anatomical images from many subjects are separately aligned to a reference atlas. Individual BOLD-weighted-to-anatomical transforms in combination with a subject's anatomical-to-atlas transform, bring all data into registration with a common atlas. However, suboptimal alignment between a subject's BOLD-weighted and anatomical images will propagate as nuisance variability that negatively affects group-level statistics for both task and resting state analyses $[6,7]$.

Significant effort has been devoted to developing methods for correcting the distortions that affect fMRI data. Currently there are two primary methods in wide use, both of which involve acquiring field map data at scan time. The first method involves acquiring a pair of EPI images with opposing phase encoding directions. Because EPI distortions mainly occur in the phase encoding direction, reversing the phase encoding direction of the acquisition also reverses the direction of the EPI distortions. By combining information from both directions,

\footnotetext{
*Corresponding author.

Email address: montez.david.f@wustl.edu (David F Montez')

${ }^{\dagger}$ These two authors contributed equally
} 
a displacement field that corrects for the underlying EPI distortion can be constructed [1]. The second field map method relies on the fact that the phase of spin-echo and gradient echo data depends on the local magnetic field and its inhomogeneities as well as the imaging echo time. By recording MR data with two different echo times and comparing the phases of the images after applying a phase unwrapping algorithm, a displacement field can be constructed that corrects for distortion caused by inhomogeneity [4]. The end product of each of these approaches is a nonlinear warp that indicates how each voxel must be displaced in order to correct for the image distortion. Without additional mitigation, the validity and quality of corrections produced by either method hinges both on the degree to which the subject moves during the acquisition of the field map data or during the time between the acquisition of the field map data and the corresponding fMRI data [8].

The need to acquire field maps introduces an additional potential failure point during data acquisition. Failure to acquire valid field map data can disqualify an entire data set from inclusion in an analysis. For instance, within the ABCD Annual Release 2.0 data set (DOI 10.15154/1503209) 341 subjects were missing valid field map data. Of the top five most downloaded fMRI data sets available on OpenNeuro.org, only one includes field map data. An effective implementation of a field map-less approach is highly desirable because it would allow correction of images for which field map information was either missing, corrupted or never collected.

Motivated by the complications of field map acquisition and the potential to reinvestigate data sets missing field maps, fMRI researchers have explored direct mapping approaches (e.g., fieldmap-less SyN-Susceptibility Distortion Correction (SDC)) in which EPI distortion is corrected by non-linearly registering a subject's distorted BOLD-weighted images to their undistorted anatomical image. Early implementations of direct mapping demonstrated that the approach could reduce distortion and improve global measures of image similarity (e.g., mutual information or squared-error) between BOLD-weighted and anatomical images [9, 10] $[11,12]$. However, further research revealed that global measures of image similarity can be an unreliable indicator of nonrigid image alignment quality [13], and that image alignment quality produced by direct mapping could be unreliable and tended to perform more poorly than high quality field map corrections [14-17]. One likely cause for the unreliability of this approach is the fact that $\mathrm{T} 1 \mathrm{w}, \mathrm{T} 2 \mathrm{w}$, and BOLD-weighted images look quite different from one another. Because the average signal intensities associated with tissues and fluids comprising the brain vary greatly across acquisition parameters, it is difficult to construct registration cost functions that can accurately reflect the true error introduced by EPI distortion [18].

We tested whether the reliability of direct mapping approaches can be improved if the undistorted anatomical images used for nonlinear warping targets were modified to increase their resemblance to the BOLD-weighted images they are meant to register. To accomplish this, we developed a modeling framework that allows us to combine information from a subject's T1w, T2w, and BOLD-weighted images in order to construct a synthetic image that has the contrast properties of a BOLD-weighted image and the undistorted geometry and high resolution $\left(\sim 1 \mathrm{~mm}^{3}\right)$ of typical $\mathrm{T} 1 \mathrm{w} / \mathrm{T} 2 \mathrm{w}$ images. We hypothesized that these synthetic BOLD-weighted images would serve as ideal targets for estimating field map corrections with 
currently available nonlinear warping software.

Here we outline a specific implementation of this general strategy for correcting EPI image distortion using synthetic images. We compare this approach to distortion correction against those produced by other commonly used MRI nonlinear registration procedures. These comparisons are performed in a subset of the Adolescent Brain Cognitive Development (ABCD) study data set [19] in order to assess performance across a variety of scanners, sites and brain geometries [20]. Additionally, we compare the performance of these different approaches in the Midnight Scan Club (MSC) data set, which consists of ten highly sampled individuals [21]. The repeated sampling of MSC subjects allowed us to assess the session-tosession variability of fMRI data corrected by Synth against other methods of EPI distortion correction. In order to facilitate the use and development of this approach by others, we provide a software package called Synth that can be used to generate synthetic images and correct fMRI distortion using the synthetic image as an alignment target. The Synth software may be used to augment existing fMRI preprocessing pipelines, or explored by researchers interested in incorporating variations on these themes into their MRI registration procedures.

\section{Methods}

\subsection{Creating an undistorted synthetic BOLD-weighted image using Synth}

Our approach to correcting EPI image distortion is first to create a synthetic BOLDweighted image based on a subject's undistorted T1w and T2w images, and then use this synthetic image as a reference for nonlinearly aligning a subjects' real BOLD-weighted image. The ideal synthetic BOLD-weighted image will have three properties: 1) it will match true BOLD-weighted data in terms of overall signal intensity, with contrast between fluids and tissue types that closely correspond to what is observed in real BOLD-weighted data; 2) It will account for the difference in spatial resolution between typically high-resolution anatomical images and typically low-resolution BOLD-weighted images and; 3) The resulting synthetic image will be affine aligned to the real image to reduce the degree to which nonlinear registration is needed to correct image discrepancies. These three demands can be expressed as the following mathematical model:

$$
\mathbf{y}=\mathrm{M}_{\gamma} \mathrm{BF} \boldsymbol{\theta}+\boldsymbol{\epsilon}
$$

Where the left hand side of the Equation (1), $\mathbf{y}$, represents a real BOLD-weighted image, and the entirety of the right hand side of Equation (1), $\mathbf{M}_{\boldsymbol{\gamma}} \mathbf{B F} \boldsymbol{\theta}$, represents an affine aligned synthetic image. Termwise, $\mathbf{M}_{\boldsymbol{\gamma}}$ corresponds to an interpolation operator representing a 12parameter affine transformation, $\boldsymbol{\gamma} ; \mathbf{B}$ is a blurring operator modeling the lower effective spatial resolution that persists when a low resolution BOLD-weighted image is upsampled to the higher resolution of its corresponding anatomical images; the matrix $\mathbf{F}$ decomposes pre-aligned $\mathrm{T} 1 \mathrm{w}$ and $\mathrm{T} 2 \mathrm{w}$ anatomical images into a set of basis vectors (e.g., radial basis functions - as in Figure 1a, or b-splines, etc.) in order to simplify the estimation of the continuous relationship between the voxel intensities of the anatomical and BOLDweighted images; $\boldsymbol{\theta}$ comprises the weights for each column of $\mathbf{F}$; and $\boldsymbol{\epsilon}$ is additive Gaussian 
noise. SynthTarget, the component of the Synth software package that we provide to solve Equation (1), does so by implementing a radial basis function (RBF) decomposition of the source $\mathrm{T} 1 \mathrm{w} / \mathrm{T} 2 \mathrm{w}$ images as $\mathbf{F}$ and then jointly optimizing the weight parameters, $\boldsymbol{\theta}$ (Figure 1b) and affine parameters, $\gamma$ (Figure 1c). The blurring operator, $\mathbf{B}$, is effected with an Epanachnikov smoothing kernel. Joint optimization to minimize mean squared error between target image and synthetic image is achieved by the method of alternating descent as illustrated in Figure 2a (See Supplemental methods for a formal account of the algorithm). When the target image, $\mathbf{y}$, is a BOLD-weighted image, and source images consist of pre-aligned $\mathrm{T} 1 \mathrm{w} / \mathrm{T} 2 \mathrm{w}$ images, SynthTarget produces a synthetic image, BF $\boldsymbol{\theta}$, based on the geometry and high resolution of the undistorted source images that retains the contrast properties of the BOLD-weighted image.

In general, the minimum number of parameters, $p$, estimated by SynthTarget is $p \geq$ $1+12+m$, corresponding to a single constant column of $\mathbf{F}$ that models baseline target image intensity; 12-parameter affine transformation, $\boldsymbol{\gamma}$; and the number of source images, $m$. This would correspond to a simple linear model with no interaction effects mapping a linear combination of source images to a target. In actual use, the total number of parameters estimated when constructing the synthetic target image, $\mathbf{y}$ will vary with the number of RBFs (intensity bins) chosen and whether the pairwise interaction effects between columns of $\mathbf{F}$ are modeled. For a hypothetical case in which the user selects as input a $\mathrm{T} 1 \mathrm{w}$ image with 10 component RBF decomposition along with a T2w image with a 5 component RBF decomposition, the number of estimated parameters will be $p=1+12+10+5=28$. The choice of RBF model, $\mathbf{F}$, is dictated by a tradeoff between computational complexity and the accuracy of the synthetic image. As the number of RBF components in $\mathbf{F}$ increases, so too do the memory requirements. For the synthetic images in the presented results, a 24 component RBF decomposition estimated at a 1mm isotropic resolution consumed $\sim 20 \mathrm{~GB}$ of RAM.

In regions of the brain with significant distortion and signal dropout affecting the EPI image, there exists no valid mapping between a subject's anatomical and BOLD-weighted images. Therefore SynthTarget also allows for the inclusion of a weight volume to reduce the contributions of these areas when estimating the parameters, $\boldsymbol{\theta}$. For the presented results, we downweighted the contributions of voxels in high-distortion areas (see Supplemental methods; Supplemental Figure 9).

\subsection{Estimating the distortion-correcting warp with Synth}

Once $\boldsymbol{\gamma}$ and $\boldsymbol{\theta}$ are estimated and the parameters are used to generate a synthetic BOLDweighted image, the next issue is correcting the EPI distortion. This can be accomplished by estimating the non-linear warp, $f$, that deforms the undistorted synthetic image to match the real BOLD-weighted image. This process is represented by Equation (2):

$$
\mathbf{y}=f\left(\mathbf{M}_{\boldsymbol{\gamma}} \mathbf{B F} \boldsymbol{\theta} ; \boldsymbol{\phi}\right)+\boldsymbol{\epsilon}
$$

Here, $\mathbf{y}$ represents the target image (e.g., a BOLD-weighed image) and $\mathbf{M}_{\gamma} \mathbf{B F} \theta$ represents the corresponding undistorted and affine aligned synthetic image of $\mathbf{y}$. The variable $\boldsymbol{\phi}$ 
bioRxiv preprint doi: https://doi.org/10.1101/2021.03.13.435270; this version posted March 15, 2021. The copyright holder for this preprint (which was not certified by peer review) is the author/funder. All rights reserved. No reuse allowed without permission.

a
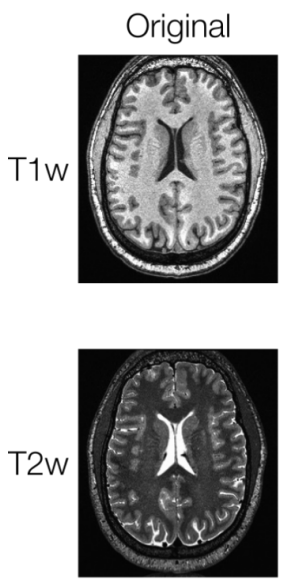

b
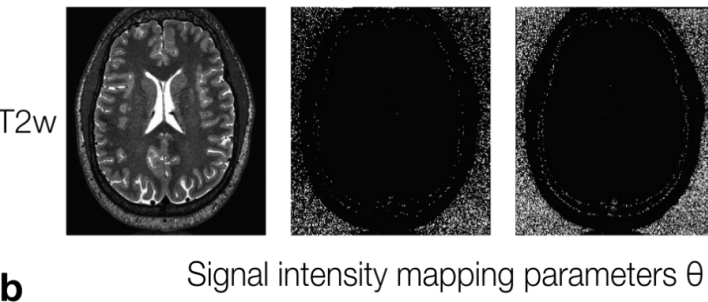
Radial basis function decomposition of T1w and T2w volumes

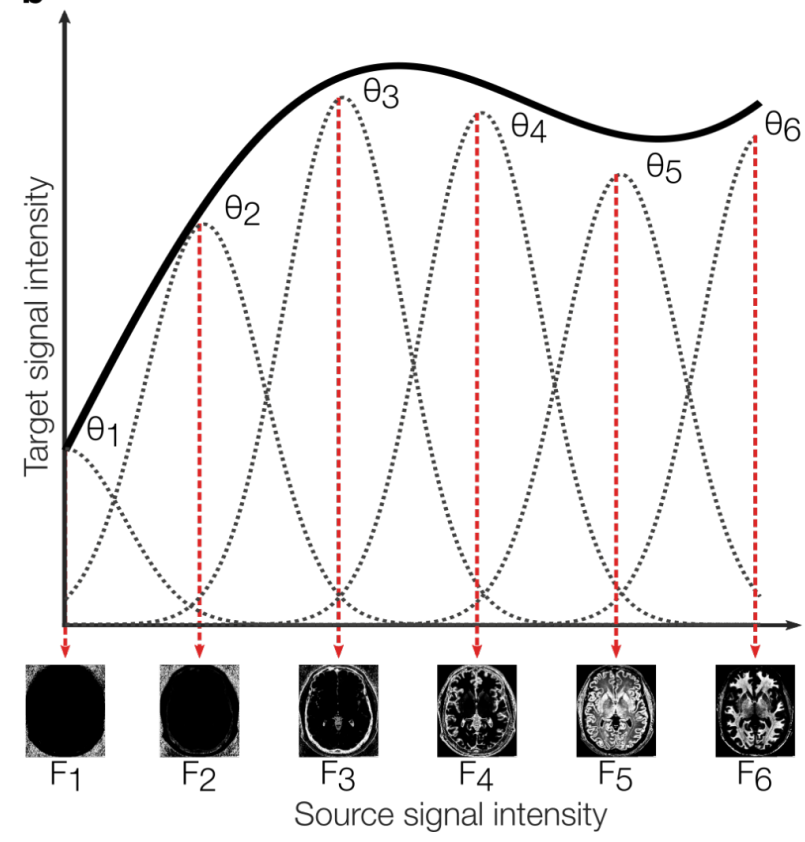

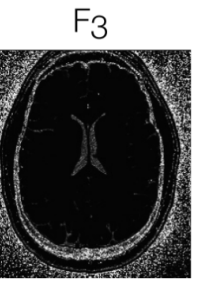

Fg
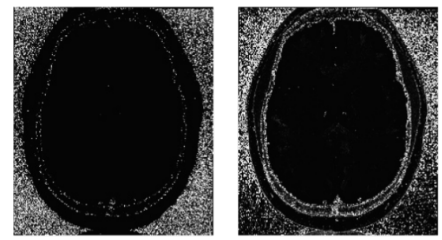

C
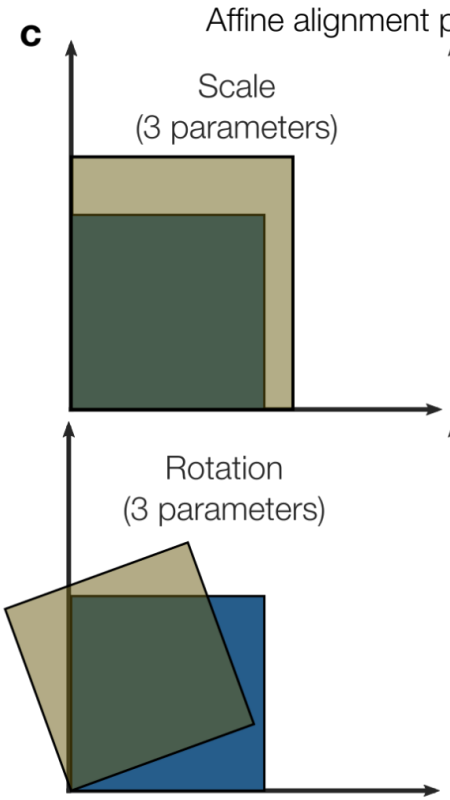

$\mathrm{F}_{4}$

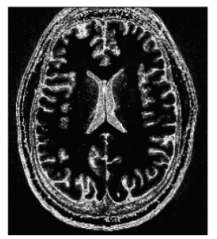

$\mathrm{F}_{10}$

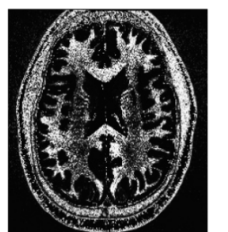

F5

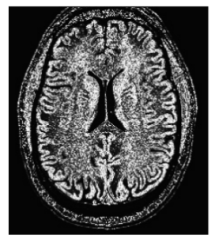

$\mathrm{F}_{11}$

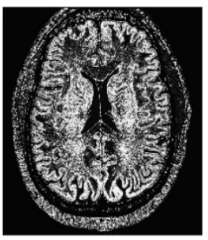

F6

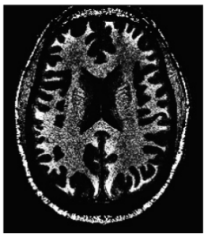

$\mathrm{F}_{12}$

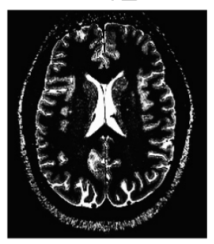

Shear

(3 parameters)

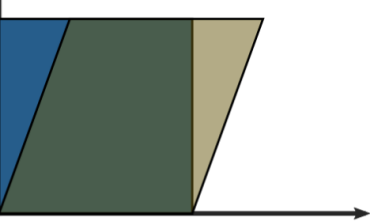

Translation

(3 parameters)

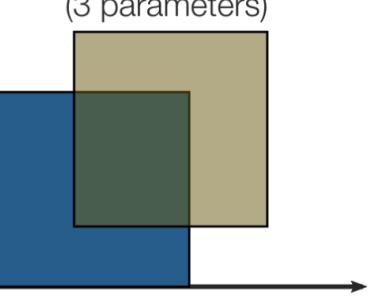

Figure 1: SynthTarget parameters (a) Illustrative radial basis function (RBF) decomposition of T1w and $\mathrm{T} 2 \mathrm{w}$ source images that comprise the columns of $\mathbf{F}$ (Equation (1)). RBF decomposition divides source images into smooth 'bins' of signal intensity so that the mapping between source and target image intensities can be estimated. Voxel intensities of a target image, e.g., a BOLD-weighted image can be modeled as linear combinations of $\mathrm{T} 1 \mathrm{w}$ and $\mathrm{T} 2 \mathrm{w}$ RBF images. For visualization purposes, we depict a six component decomposition of the images; Synth Target allows for an arbitrary degree of image decomposition to maximize flexibility in modeling target images with different contrast properties. (b) A portion of a hypothetical nonlinear relationship between voxel intensity values observed between source image RBF components (e.g., T2w; x-axis) and voxel intensity values observed in a target image (e.g., BOLD-weighted). This image depicts the $\mathbf{F} \boldsymbol{\theta}$ portion of Equation (1). (c) The parameter set $\gamma$ corresponds to the standard twelve affine parameters, scale; shear; rotation; and translation that affect the physical alignment between target and source images. SynthTarget jointly optimizes $\boldsymbol{\theta}$ and $\boldsymbol{\gamma}$. 
represents the underlying parameterization of the nonlinear transformation, $f$. The inverse of the estimated nonlinear warp, $f^{-1}$, may then be computed. $f^{-1}$ is analogous to a traditional field map correction and maps voxels in a distorted image back to their correct locations.

In principle, many of the pre-existing nonlinear registration utilities are suitable for estimating $f$ and $f^{-1}$, owing either to their methods of diffeomorphic warp construction which guarantee the existence of an invertible warp - as is the case with AFNI's 3dQwarp [22] and ANTs SyN [23] — or their ability to project a potentially non-invertible warp onto a "nearest invertible" space - as is the case with FSL's FNIRT [24] . For the results presented here and in the reference preprocessing scripts associated with this manuscript, we used the ANTs SyN algorithm with a local cross correlation metric for estimating all non-linear warps (see Supplemental methods for details). ANTs SyN was chosen for its reliable high performance in non-linear warp estimation [17, 25, 26].

After correcting the EPI image distortion by applying $f^{-1}$ to the real BOLD-weighted data, there is improved geometric correspondence between anatomical images and functional images. Because the quality and accuracy of a synthetic BOLD-weighted image depends on how closely registered the anatomical and true BOLD-weighted images are, modest improvements to the synthetic image can be achieved by re-running SynthTarget using the newly corrected BOLD-weighted image as a target. Therefore, in our reference processing scripts and for the results presented here, we refined the distortion-correcting warp over three iterations by re-estimating an improved synthetic image based on the warp produced during the prior iteration, then updating subsequent warp estimates using the improved synthetic image (see Supplemental methods for formal algorithmic details). The complete procedure is implemented by the component of the Synth software package called SynthUnwarp.

\section{Description of data sets and processing}

We assessed the quality of distortion corrections produced by direct mapping to Synthgenerated synthetic images in two different contexts representing important use cases for fMRI data. First, we examined a subset of 100 subjects selected randomly from the Adolescent Brain Cognitive Development (ABCD) study data set (median age: 9.85 years; min: 9 years; max: 11 years). Our random sample included data acquired using GE, Philips and Siemens scanners (see Supplemental Table 1). Second, we evaluated Synth's performance on the Midnight Scan Club (MSC) data set, which consists of resting state fMRI scans acquired from 10 subjects on 10 separate occasions (300 minutes of resting state fMRI data/subject). The MSC precision functional mapping (PFM) [27-32, 32-34] data set allowed us to assess session-to-session reliability of EPI distortion correction schemes across multiple sessions for the same subject. Importantly, image acquisition parameters for ABCD and MSC data sets differ significantly allowing us to assess the performance of Synth distortion correction on images with a range of image contrast and levels of detail. Image acquisition parameters have been reported in detail elsewhere [21,35].

We compared the effectiveness of this approach against four widely used distortion correction algorithms. Three of these approaches rely on separately acquired field map data (FSL fugue; FSL topup; and AFNI's 3dQwarp) while the third approach implements an 


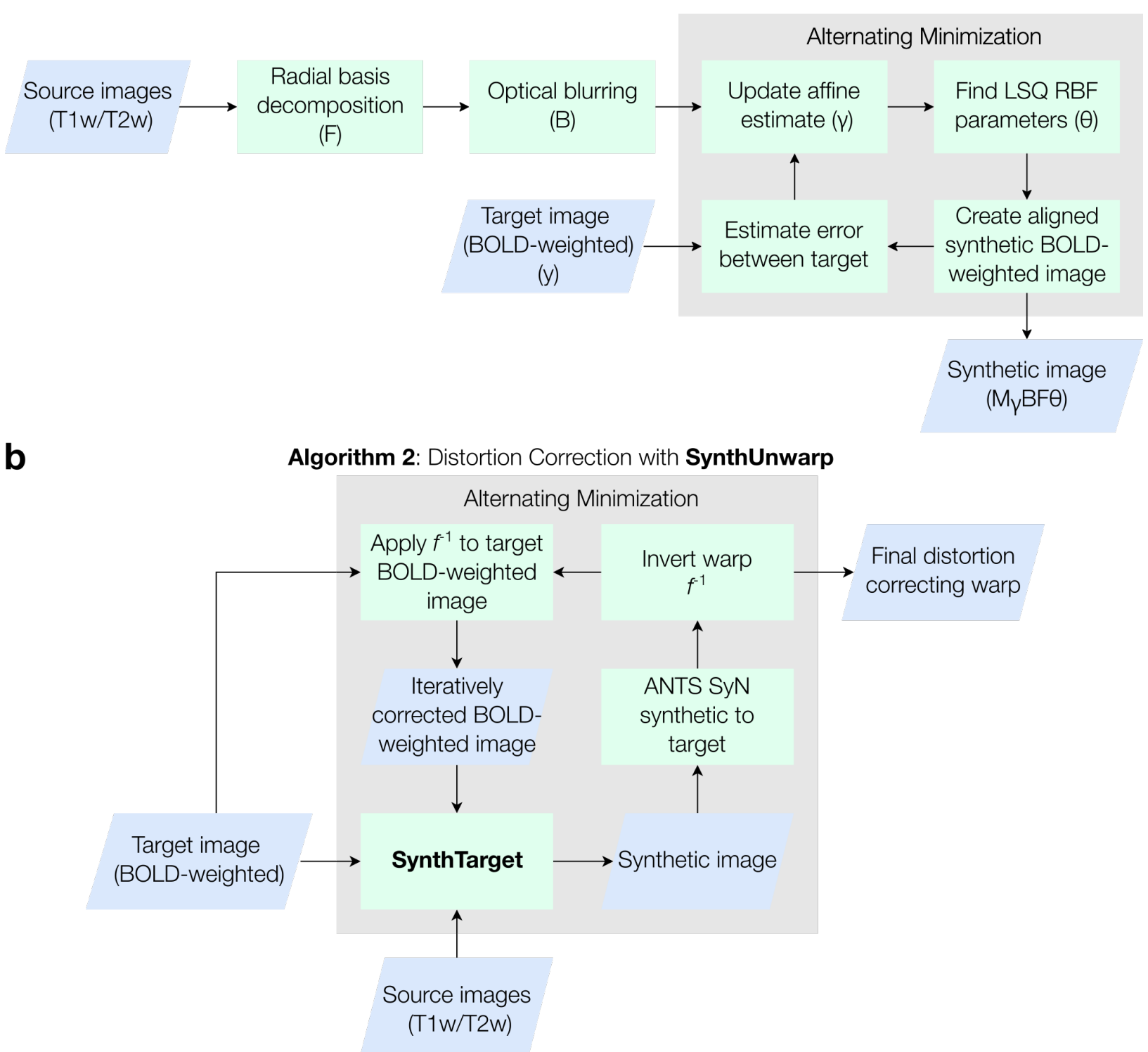

Figure 2: Flow chart descriptions of synthetic image creation and EPI distortion correction using Synth . (a) Process for estimating a synthetic image using SynthTarget. Source images (in this case $\mathrm{T} 1 \mathrm{w} / \mathrm{T} 2 \mathrm{w}$ images) are decomposed into radial basis function (RBF) components, $\mathbf{F}$, and blurred with a blurring operator, $\mathbf{B}$. The affine operator, $\mathbf{M}_{\boldsymbol{\gamma}}$, and RBF weights, $\boldsymbol{\theta}$ are iteratively optimized in an alternating minimization scheme (gray process box) to find the minimum mean squared error between the model (Equation (1)) and the target image. In alternating minimization, parameters are updated repeatedly in an alternating sequence in which one parameter is held fixed and the other is optimized. The optimal parameters correspond to the synthetic image, $\mathbf{B F} \boldsymbol{\theta}$, aligned to the target image, $\mathbf{y}$, using the optimal affine operator, $\mathbf{M}_{\gamma}$. (b) Process for distortion correction using SynthUnwarp. The displacement field, $f$, that aligns the initial synthetic image to the target image is estimated using the SyN algorithm. The resulting displacement field, $f$, is inverted to produce the distortion correcting warp, $f^{-1}$, which reduces the target image distortion. This improves the correspondence between the target and synthetic images, and by proxy, the source images, allowing for improved estimates of a new synthetic image. The distortion correcting warp, $f^{-1}$, is updated in an alternating minimization scheme, during which the synthetic image is refined after each improved estimate of $f^{-1}$. 
alternative field map-less distortion correction method (ANTs $S y N-S D C$ ). FSL field map corrections were constructed using either fugue (used for double echo field maps acquired in the MSC data set) or topup (used for opposite direction phase encoded field maps in the ABCD data set). The resulting field map corrections were passed into epi_reg [36], a standard pipeline that implements a boundary based registration method, used to simultaneously unwarp the functional image and align the functional to the anatomical image [37]. In AFNI, the distortion correction is estimated from opposite direction phase encoded field maps using a "meet in the middle" nonlinear warp estimation implemented in 3dQwarp [22]. This estimated warp is used to correct the image and combined with a separately estimated rigid body transform to align the functional to the anatomical image. Finally, we assessed the $S y N-S D C$ field map-less method that corrects distortion by non-linearly aligning the subject's EPI and anatomical images while constraining allowable warps to regions known to be strongly affected by distortion $[16,17,38]$.

Performance of the different distortion correction approaches was assessed in matched data sets, thus all statistical comparisons were performed within a multi-level modeling framework (fitlme, MATLAB). For these analyses, the metrics produced by the Synth-based registration pipeline were modeled as the baseline and differences in performance metrics associated with other approaches were modeled with main effect factors. To account for subject-specific variability, independent of registration approach, subject identity was modeled as a random effect. The focus of this manuscript is assessing the quality of Synth-based registration in relation to other current approaches, not an exhaustive cross-comparison of all approaches, and we report only the related statistical contrasts here.

\section{Results}

\subsection{Synthetic images are more similar to BOLD-weighted images than both T1w and T2w images}

Motivated by the idea that undistorted synthetic BOLD-weighted images may be a more suitable reference for correcting distorted fMRI data than $\mathrm{T} 1 \mathrm{w} / \mathrm{T} 2 \mathrm{w}$ images, we set out to determine whether Synth could produce synthetic images with contrast properties that were quantifiably more similar to real BOLD-weighted images than the subjects' $\mathrm{T} 1 \mathrm{w}$ or $\mathrm{T} 2 \mathrm{w}$ images despite having identical underlying geometry. Balancing computational costs and synthetic image accuracy, we found that a model that included a 12-component radial basis function (RBF) decomposition of the $\mathrm{T} 1 \mathrm{w} / \mathrm{T} 2 \mathrm{w}$ source images was sufficient to model an effective synthetic BOLD-weighted image for the MSC data set. For the ABCD data set, a more complex 12-component RBF model with pair-wise $\mathrm{T} 1 \mathrm{w} / \mathrm{T} 2 \mathrm{w}$ interaction terms was required to create synthetic images with a similar level of BOLD-weighted contrast similarity (Figure 3). To quantify image similarity, we registered pre-aligned $\mathrm{T} 1 \mathrm{w} / \mathrm{T} 2 \mathrm{w}$ images as well as their associated synthetic images to their corresponding real BOLD-weighted images using identical affine transformations $\left(M_{\gamma}\right)$. Reasoning that contrast properties are reflected in the spatial frequency content of an image, and that the power spectra of an image is largely invariant to translational differences (a property of the Fourier transform), we converted the identically aligned T1w, T2w, synthetic, and BOLD-weighted images (Figure 3a) into their 
respective power spectra representations and then normalized them to unit magnitude to allow for comparison across image types. For each session of data, we quantified contrast similarity between the subject's BOLD-weighted image and each of their T1w, T2w and synthetic images by computing the dot product of their normalized power spectra vectors. For this contrast similarity metric, values range between 0 and 1, where a value of 1 indicates identical images, potentially differing by a small translation.

Regardless of imaging modality, the overall spatial structure observed in brain images is similar. It was therefore unsurprising to observe high contrast similarity values between the BOLD-weighted images and their associated T1w/T2w and Synth images in general. Across both data sets, however, contrast similarity was highest between the BOLD-weighted and Synth images (Figure 3b,c). In the ABCD data set, for instance, we observed that contrast similarity was lowest between the BOLD-weighted and T1w images $(\mu=0.85 \sigma=0.034)$, while contrast similarity was somewhat higher between BOLD-weighted and T2w images $(\mu=0.92, \sigma=0.015)$. Contrast similarity between the BOLD-weighted and Synth images was observed to be both higher and less variable $(\mu=0.96, \sigma=0.007)$. Mixed-effects model comparisons revealed that the differences between Synth contrast similarity and T1w/T2w contrast similarity were both significant: (T1w-Synth $=-0.11 ; \mathrm{p}=3.24 \mathrm{e}-111 ; \mathrm{t}=-36.3 ; \mathrm{df}=297$ ) and $(\mathrm{T} 2 \mathrm{w}-$ Synth $=-0.03 ; \mathrm{p}=3.6 \mathrm{e}-23 ; \mathrm{t}=-10.8 ; \mathrm{df}=297)$. In the MSC data set, we observed that neither $\mathrm{T} 1 \mathrm{w}(\mu=0.85, \sigma 0.027)$ nor $\mathrm{T} 2 \mathrm{w}(\mu=0.86, \sigma=0.018)$ images exhibited consistently greater contrast similarity to BOLD-weighted images across subjects. This ambiguity may be a consequence of the lower native resolution of the MSC BOLD-weighted images compared to the ABCD BOLD-weighted images $\left(4 \mathrm{~mm}^{3}\right.$ vs $\left.2.4 \mathrm{~mm}^{3}\right)$. Uniformly, though, Synth images exhibited the greatest contrast similarity $(\mu=0.96, \sigma=0.005)$. Mixed-effects model comparison verified what was plainly visible upon inspection of the images (Figure 3ac): $(\mathrm{T} 1 \mathrm{w}-$ Synth $=-0.10 ; \mathrm{p}=3.3 \mathrm{e}-186 ; \mathrm{t}=-69.8 ; \mathrm{df}=297)$ and $(\mathrm{T} 2 \mathrm{w}-$ Synth $=-0.96 ; \mathrm{p}=2.8 \mathrm{e}-177$; $\mathrm{t}=-64.8 ; \mathrm{df}=297)$.

\subsection{Nonlinear registration to synthetic images improves correspondence to anatomical im- ages}

Previous field map-less approaches for correcting distortion by directly mapping EPI images to their associated anatomical images have been unreliable [14-16]. This unreliability takes the form of anomalous blurring and distortion in the corrected EPI image, despite the large number of degrees of freedom the nonlinear warp provides for reducing the magnitude of the error term between two images (e.g., see Fig. 1 in [15]) Therefore, having determined that Synth reliably produces undistorted images with BOLD-weighted contrast, we set about assessing whether fMRI data corrected by direct mapping to synthetic images exhibit residual distortion affecting the brain.

First, we generated a distortion correcting warp by inverting the transformation, $f$ (Equation (2)), that maps the undistorted synthetic image onto the true BOLD-weighted image. We then applied this inverted transformation to the true BOLD-weighted data and examined the quality of the registration between the resulting corrected BOLD-weighted images and the corresponding subject's anatomical image. We observed that this procedure visibly improved upon affine-only registration between the BOLD-weighted and anatomical images 
a
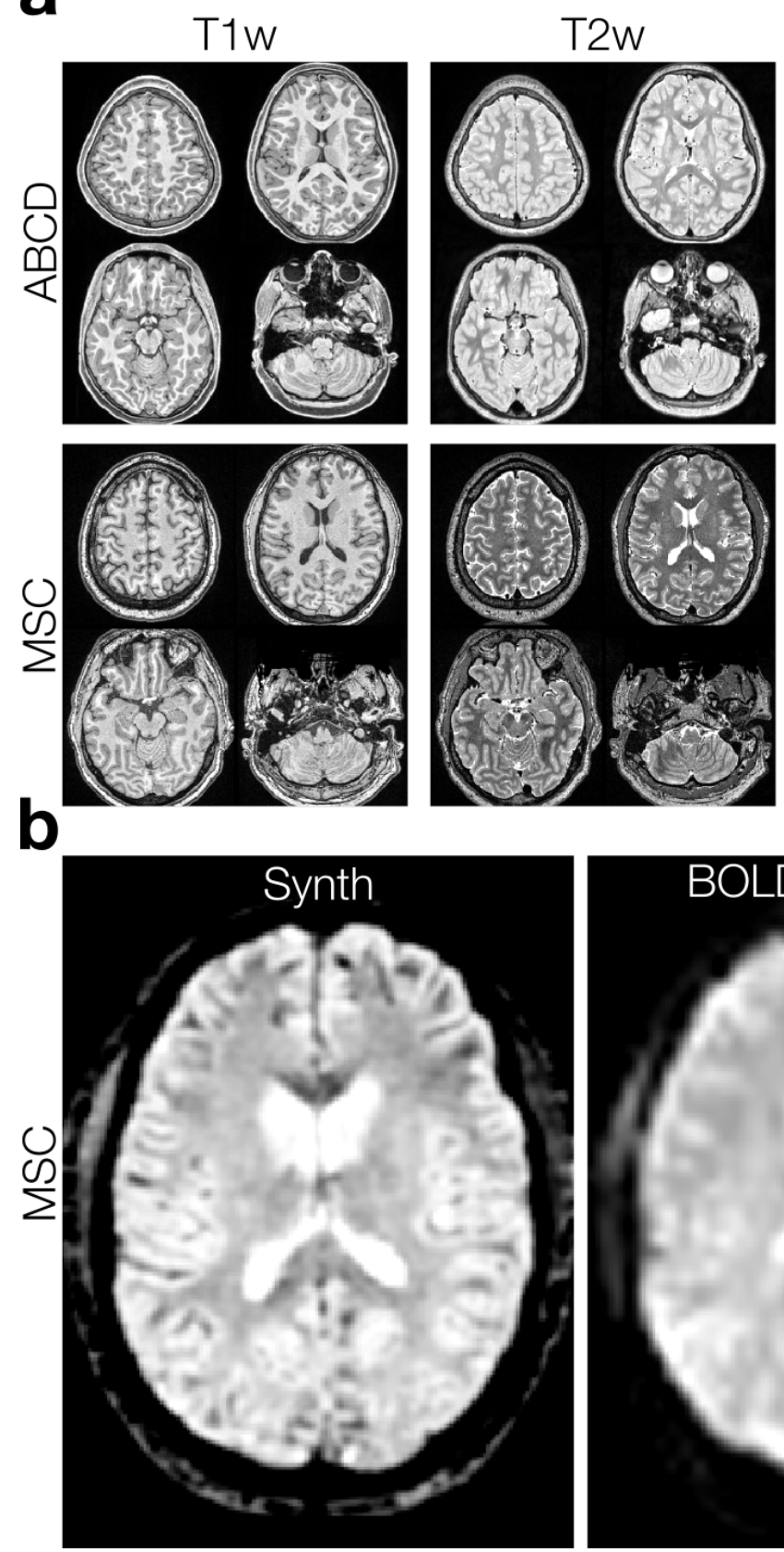
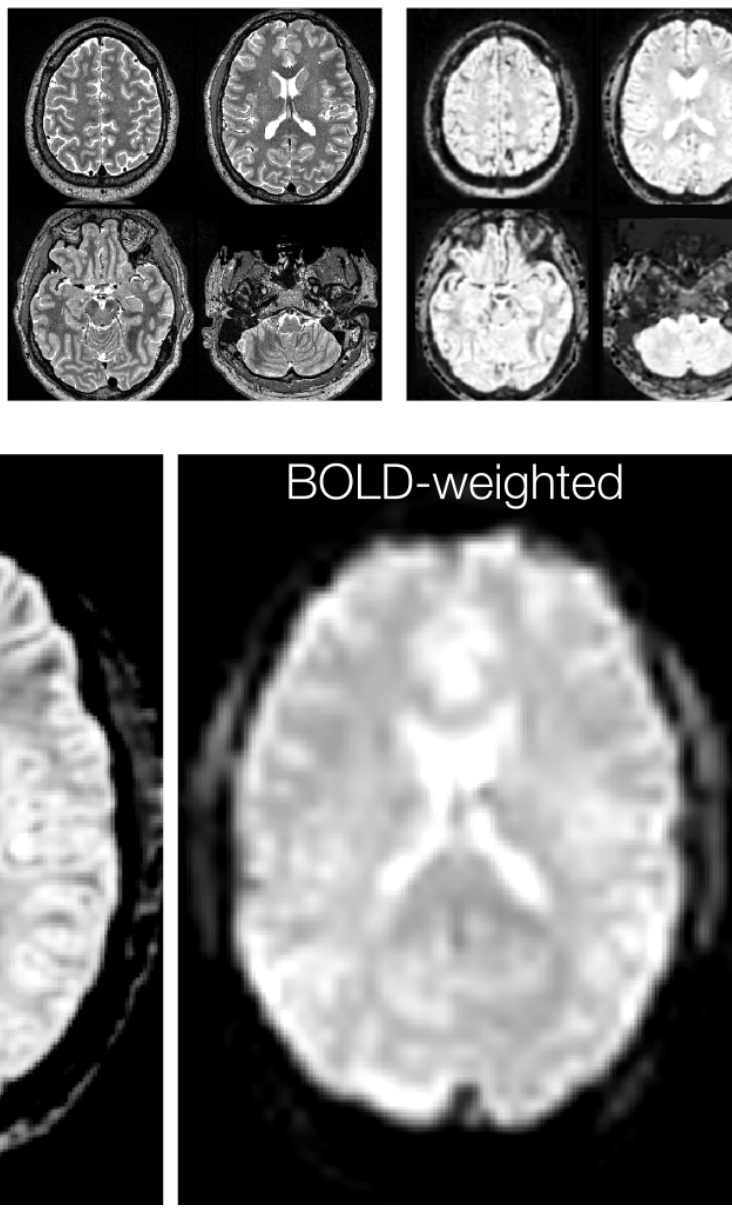

Synth
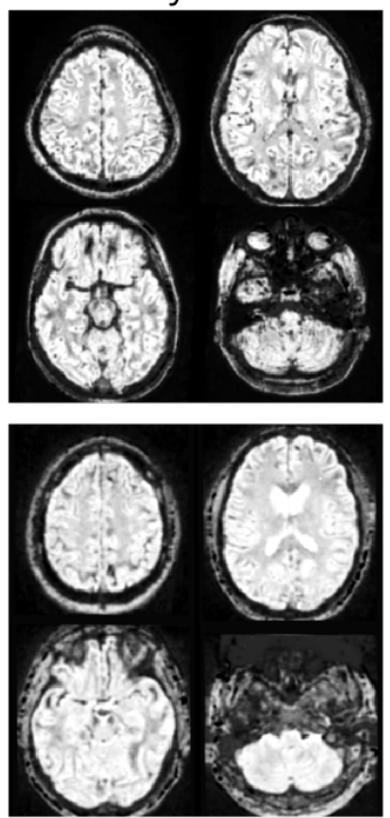

\section{C}

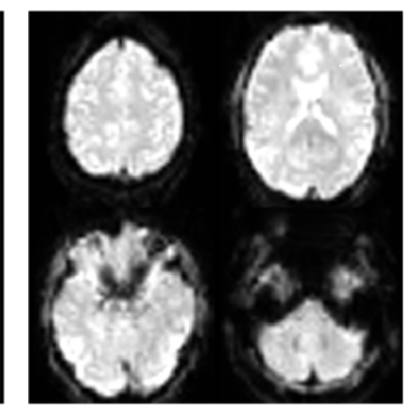

Synth T1w T2w

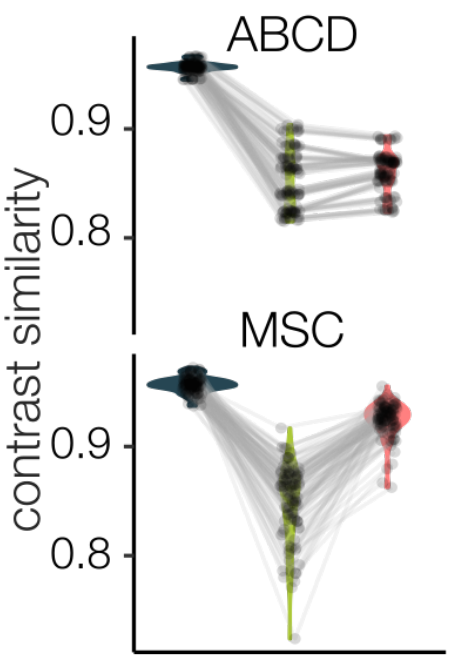

Figure 3: Image contrast similarity between T1w, T2w, Synth and BOLD-weighted images. (a) A visual comparison of contrast similarity between a representative subject's T1w, T2w, synthetic, and corresponding affine aligned BOLD-weighted images (from left to right). Top row depicts an arbitrarily selected ABCD subject, the bottom row an arbitrarily selected MSC subject. The greatest visual similarity is between the synthetic image and the true BOLD-weighted image. (b) Enlarged view of an axial slice of the MSC subject's synthetic (left) and real BOLD-weighted (right) images. (c) Violin plots depicting average contrast similarity between BOLD-weighted images and associated anatomical and synthetic images across 100 subjects from the ABCD data set (top). Overall contrast similarity between a true BOLD-weighted image and associated anatomical and synthetic functional images for each subject in the MSC data set (bottom). Each subject contributed 10 sessions of data. 
with little anomalous distortion visibly affecting the brain (Figure 4). Improved registration was apparent at both large and small spatial scales. Large regions of misregistration, corresponding to relatively low spatial frequency distortions, clearly visible in the affine-only aligned images, were largely corrected (Figure 4a-c). Finer details captured by higher spatial frequency components of the warp, such as the registration of individual sulcal folds, were also visibly improved (Figure 4d-f).

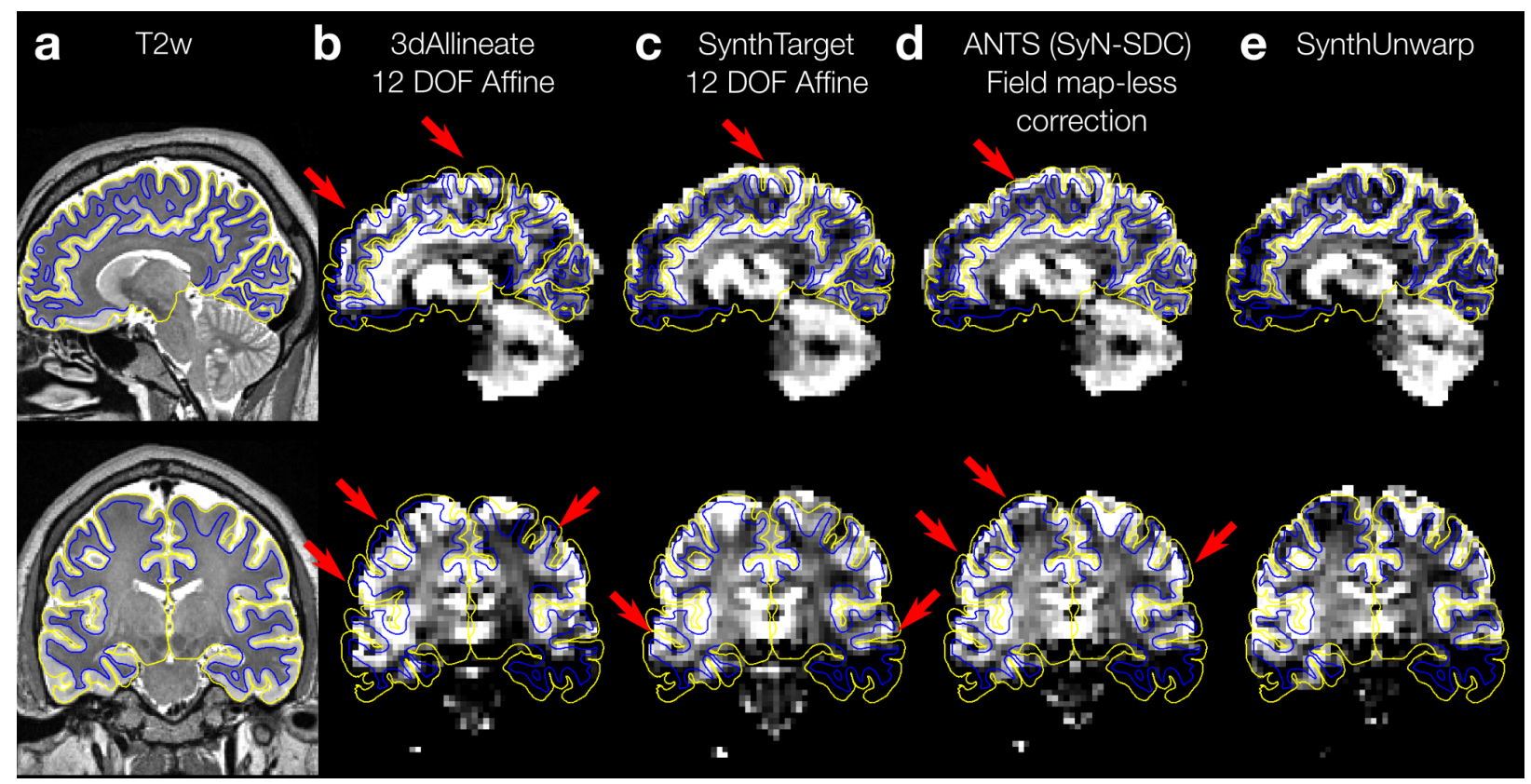

Figure 4: Influence of using synthetic images on cortical alignment using affine and non-linear registration. Matched sagittal (top row) and coronal (bottom row) slices depicting T2w and BOLDweighted images acquired from a representative subject drawn from the MSC data set (MSC01). (a) Reference T2w image overlaid with FreeSurfer derived white matter (blue) and pial (yellow) boundaries. (b) $12 \mathrm{DOF}$ affine registration to the $\mathrm{T} 2 \mathrm{w}$ image (2nd column) results in poor alignment of the anterior half of the brain (red arrows) (c) 12 DOF alignment to synthetic target visual improves overall correspondence to $\mathrm{T} 2 \mathrm{w}$ image, however local misalignment of sulci and gyri remain (red arrows) (d) Field map-less nonlinear alignment with ANTs SyN-SDC procedure improves alignment of sulci and gyri at the cost of an anomalous warp placing the subarachnoid cerebrospinal fluid into the gray matter regions between white matter and pial boundaries (e) Nonlinear alignment to a synthetic image resulting in high quality global and local alignment.

\subsection{Nonlinear registration to synthetic images improves global measures of anatomical and functional image alignment}

To characterize the performance of Synth relative to other common distortion correction methods (AFNI, FSL, ANTS), we created matched ABCD and MSC data sets identically processed with the exception of the approach used for fMRI distortion correction (see Supplemental Methods) and computed a series of global and local alignment quality metrics. We compared the synthetic image alignment performance against widely used utilities from AFNI (3dAllineate + 3dQwarp), FSL (topup/fugue + epi_reg) and ANTs (fieldmap-less 
SyN-Susceptibility Distortion Correction $(S D C)$ ) software packages [1, 16, 17, 37-39]. Field maps in the MSC data set were acquired using the phase difference of differing echo times approach. Owing to the incompatibility of the underlying data and the field map estimation approach implemented by the software, we could not assess 3dQwarp performance on the MSC data set and therefore only compared Synth's performance to FSL's epi_reg and ANTs $S y N S D C$. In order to ensure that apparent differences in performance were not the result of differences in underlying interpolation algorithms, all transformations were converted to AFNI compatible formats, composed and applied using the 3dNwarpApply utility.

There is currently no consensus on what single global metric best quantifies alignment quality. Many metrics used to measure alignment quality tend to bear some relation to the cost functions that are used during registration and therefore directly introduce a risk of confounding circularity. Thus, we computed several kinds of metrics comparing the similarity of different features of the field map corrected BOLD-weighted images to their associated anatomical images. This included:

1. Normalized mutual information (NMI) shared between the time-averaged BOLDweighted image from each session and its associated $\mathrm{T} 1 \mathrm{w} / \mathrm{T} 2 \mathrm{w}$ anatomical images (e.g., T1w-BOLD NMI, T2w-BOLD NMI), which assesses global image similarity (Figure $5 \mathrm{a})$.

2. The correlation coefficient between the gradient magnitude images of the time-averaged BOLD-weighted image and T1w/T2w images, which quantifies the degree of alignment between high-contrast edges found in two images (Figure 5b).

3. Three segmentation metrics designed to assess the degree to which distinct areas of the anatomical images correspond to distinct ranges of voxel intensity values within the aligned BOLD-weighted images. For instance, in most BOLD-weighted images, gray matter voxels tend to have higher signal intensity than white matter voxels. Proper registration to an anatomical image should make the distributions of BOLD-weighted voxel intensities associated with white (WM) and gray matter (GM) (as determined by segmentation of the anatomical images) more separable. Therefore, the first of the segmentation metrics, $\mathrm{AUC}_{g w}$ is defined as the area under the receiver operating characteristic (ROC) curve of the distributions of directly adjacent gray and white matter signal intensities - where 'adjacent' refers to the set of all gray matter voxels that share a face with a white matter voxel and vice versa. In a similar vein, $\mathrm{AUC}_{v w}$ assesses the difference between the typically high intensity voxels representing the ventricles and low intensity voxels representing adjacent white matter. Lastly, because brain matter BOLD-weighted voxels tend to have higher signal intensity than voxels representing the areas immediately exterior to the brain volume, we computed $\mathrm{AUC}_{i e}$, to quantify the overall registration of brain and brain-exterior boundaries (Figure 5c).

In both $\mathrm{ABCD}$ and MSC data sets, Synth registration tended to produce the BOLDweighted images with the highest registration quality metrics (Figure 5). Here, we outline Synth registration performance for each metric described above as compared to the highest performing competitor. Full statistical tables comparing all methods are included in Supplemental materials. In the ABCD data set, Synth aligned BOLD-weighted images shared 


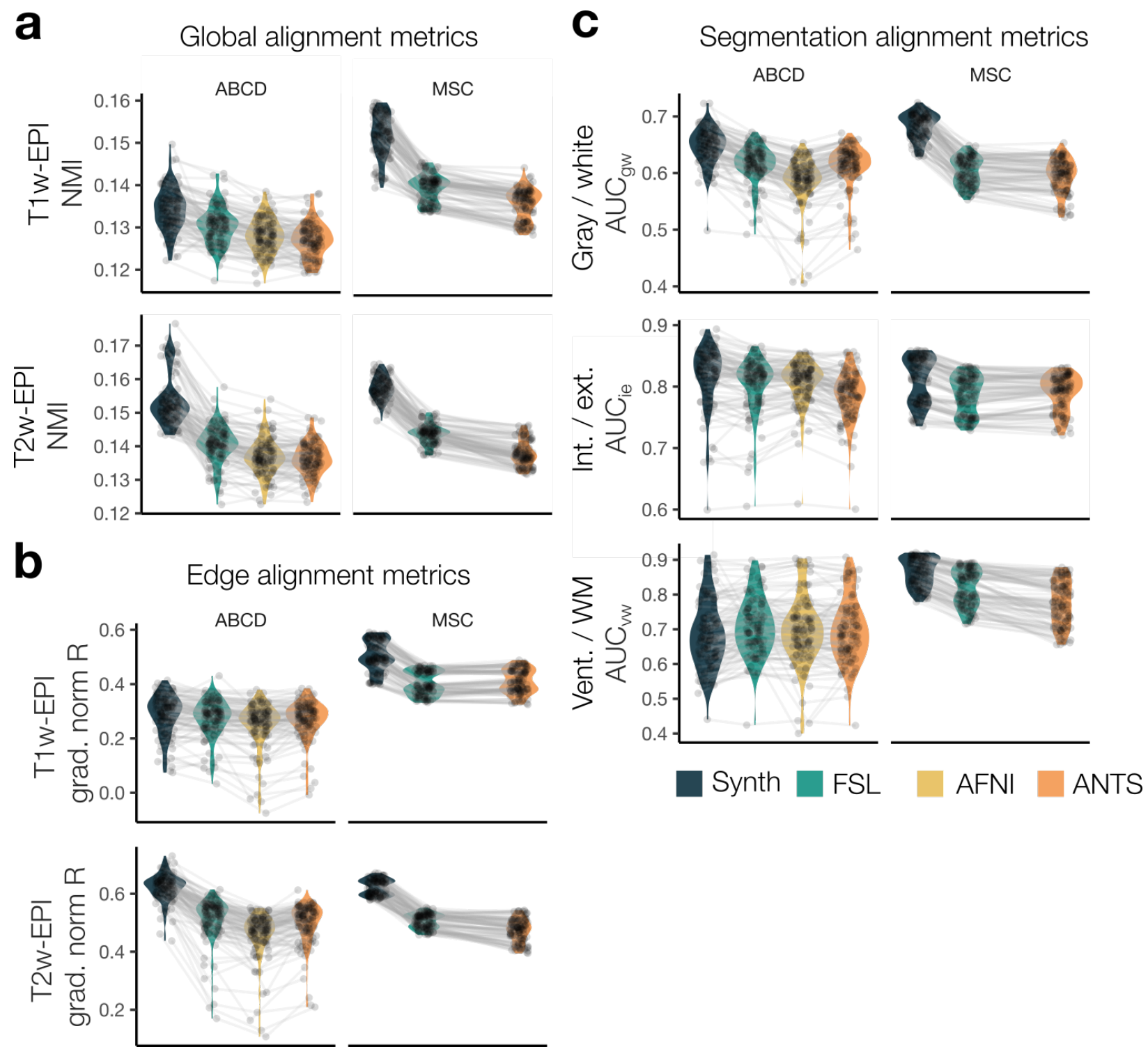

Figure 5: Comparison of alignment metrics across registration pipelines for ABCD and MSC data sets. Violin plots depicting (a) Normalized mutual information (NMI) shared between the registered BOLD-weighted images and their associated $\mathrm{T} 1 \mathrm{w} / \mathrm{T} 2 \mathrm{w}$ anatomical images to assess global similarity. (b) Correlation coefficient between the gradient magnitude images for BOLD-weighted and $\mathrm{T} 1 \mathrm{w} / \mathrm{T} 2 \mathrm{w}$ images for a metric of edge alignment. (c) Segmentation alignment metrics that quantify the separability of anatomical features in BOLD-weighted data based on the FreeSurfer segmentation of their anatomical T1w images. 
greater normalized mutual information (NMI) with both T1w (FSL-Synth $=-4 \mathrm{e}-3 ; \mathrm{p}=5.78 \mathrm{e}-$ $32 ; \mathrm{t}=-12.88 ; \mathrm{df}=396)$ and $\mathrm{T} 2 \mathrm{w}(\mathrm{FSL}-$ Synth $=-1.3 \mathrm{e}-2 ; \mathrm{p}=8.85 \mathrm{e}-93 ; \mathrm{t}=-27.23 ; \mathrm{df}=396)$ images than the next highest registration procedure (FSL topup). Synth alignment produced BOLDweighted images with the highest NMI shared between $\mathrm{T} 1 \mathrm{w} / \mathrm{T} 2 \mathrm{w}$ images in the MSC data set as well (FSL-Synth $=-4 \mathrm{e}-3 ; \mathrm{p}=5.78 \mathrm{e}-32 ; \mathrm{t}=-12.88 ; \mathrm{df}=396$ ).

We obtained a similar pattern of results when comparing the registration of BOLDweighted and anatomical image edges by correlating their gradient norm magnitude images. In the ABCD data set, Synth registration produced images with significantly greater alignment metrics than all other methods. T1w: (FSL-Synth $=-1 \mathrm{e}-2 ; \mathrm{p}=0.003 ; \mathrm{t}=-3.1 ; \mathrm{df}=396)$; T2w: (FSL-Synth $=-0.1 ; \mathrm{p}=2.28 \mathrm{e}-75 ; \mathrm{t}=-23.1 ; \mathrm{df}=396)$. Although Synth registration also produced the greatest edge alignment metrics in the MSC data set, its closest competitor varied by modality. For BOLD-T1w edge registration, ANTS produced the best of the non-Synth metrics, however it was still significantly lower than Synth registration (ANTsSynth $=-0.1 ; \mathrm{p}=3.85 \mathrm{e}-154 ; \mathrm{t}=-53.32 ; \mathrm{df}=297)$. For $\mathrm{T} 2 \mathrm{w}$ images in the MSC data set, FSL was once again the closest competitor, (FSL-Synth $=-0.12 ; \mathrm{p}=1.15-156 ; \mathrm{t}=-54.9 ; \mathrm{df}=297$ ).

On image segmentation alignment metrics, Synth performed similarly well: $\mathrm{AUC}_{g w}$ was found to be greater for Synth-aligned images in both ABCD (FSL-Synth=-0.03; p=1.16e$33 ; \mathrm{t}=-13.31 ; \mathrm{df}=396)$ and MSC (FSL-Synth $=-0.08 ; \mathrm{p}=3.19 \mathrm{e}-144 ; \mathrm{t}=-49 ; \mathrm{df}=297)$ data sets. This result indicates improved gray matter to gray matter correspondence between EPI and anatomical images and a reduction in partial voluming effects due to misregistration. Synthaligned images also exhibited greater $\mathrm{AUC}_{b e}$ in both $\mathrm{ABCD}$ (AFNI-Synth $=-0.01 ; \mathrm{p}=1.2 \mathrm{e}-5$; $\mathrm{t}=-4.43 ; \mathrm{df}=396)$ and MSC (ANTs-Synth=-0.021; $\mathrm{p}=2.32 \mathrm{e}-49 ; \mathrm{t}=-18 ; \mathrm{df}=297$ ) data sets than the nearest competitor pipeline, AFNI and ANTs respectively.

One exception to the overall trend of improved segmentation alignment metrics produced by Synth registration was observed in the ABCD data set. Here, we found that the FSL pipeline produced greater $\mathrm{AUC}_{v w}$ values (FSL-Synth $=0.023 ; \mathrm{p}=5.7 \mathrm{e}-5 ; \mathrm{t}=4.1 ; \mathrm{df}=396$ ). However, the ventricle alignment metrics of Synth aligned images did not differ significantly compared to images produced by either ANTs $(\mathrm{p}=0.1)$ or AFNI $(\mathrm{p}=0.15)$ pipelines. In contrast, Synth registration produced EPI images with the $\mathrm{AUC}_{v w}$ values that were significantly higher than the nearest competitor, FSL (FSL-Synth $=-0.05 ; \mathrm{p}=1.1 \mathrm{e}-42 ; \mathrm{t}=-16.2 ; \mathrm{df}=297$ ) in the MSC data set.

\subsection{Nonlinear registration with Synth improves local measures of anatomical and functional image alignment}

Often, approaches to image registration rely on minimizing or maximizing a global distance or similarity metric (e.g., least-squares or mutual information) computed across an entire image volume. These metrics can be strongly influenced by the presence of intensity bias fields, which are low spatial frequency modulations of image intensity often caused by suboptimal subject placement or poor shimming. Additionally, some experimental evidence has suggested that inaccurate registration can still produce high values for many global measures of image alignment [13]. We therefore sought to compare the performance of each approach to EPI distortion correction with respect to a measure of local image similarity that would be less sensitive to these effects. 
To do this, we implemented a 'spotlight' analysis examining $7 \mathrm{x} 7 \mathrm{x} 7$ voxel regions $(3 \mathrm{~mm}$ isotropic voxels) of the BOLD-weighted images produced by each method and quantifying its similarity to the corresponding region of the subject's T1w and T2w images with an $\mathrm{R}^{2}$ metric. Image registration quality near gray matter is typically of greatest interest, hence we summarized local T1w-BOLD and T2w-BOLD similarity by computing the average spotlight $\mathrm{R}^{2}$ across all gray matter voxels, as labeled by the subject's FreeSurfer segmentation (Figure 6a). In the ABCD data set, we observed that Synth and FSL registration pipelines produced similar quality of local registration to the subjects' $\mathrm{T} 1 \mathrm{w}$ image, with both leading AFNI and ANTs pipelines (FSL-Synth $=-0.002 ; \mathrm{p}=0.15 ; \mathrm{t}=-1.44 ; \mathrm{df}=396$ ). Average local similarity between BOLD-weighted and T2w images was greatest for Synth registered images with FSL producing the second highest metrics (FSL-Synth=-0.11; $\mathrm{p}=2.3 \mathrm{e}-75 ; \mathrm{t}=-23.1$; $\mathrm{df}=396)$. We observed a more uniform result in the MSC data set: Here, Synth registration produced BOLD-weighted images with the greatest average local similarity for both the associated T1w (FSL-Synth=-0.06; $\mathrm{p}=4.9 \mathrm{e}-174 ; \mathrm{t}=-63 ; \mathrm{df}=297)$ and T2w $(\mathrm{FSL}-$ Synth $=-0.12$; $\mathrm{p}=1.13 \mathrm{e}-170 ; \mathrm{t}=-61 ; \mathrm{df}=297)$ images.

We constructed 'winner-take-all' maps that depict, for each voxel, which registration method produced the greatest average local similarity $\left(7 \times 7 \times 7\right.$ voxel spotlight $\left.\mathrm{R}^{2}\right)$ between $\mathrm{T} 1 \mathrm{w}$ and $\mathrm{T} 2 \mathrm{w}$ anatomical images (Figure $6 \mathrm{~b}$ ). We observed that the distortion correction approach that produced the greatest local BOLD-T1w similarity depended on the data set and the specific regions of the brain under consideration. In both the ABCD and MSC data sets, registering to Synth's synthetic image produced the highest local BOLD-T1w similarity across the greatest percentage of voxels.

\subsection{Nonlinear registration with Synth improves intrasubject consistency across sessions}

We used the MSC data set to investigate how different distortion correction procedures affect the inter-session stability of measures of fMRI data quality. Here, we hypothesized that improved anatomical-to-functional registration would result in greater consistency (equivalently, less variability) between the resulting mean functional volumes from each session for a given individual. To quantify registration related variability, we extracted the region of the aligned mean BOLD volumes containing just brain matter (as classified by the subject's FreeSurfer segmentation) from each session. Then, for each subject, we computed the average linear correlation coefficient across all pairs of sessions (Figure 7a).

Owing to the general stability of a given subject's brain geometry on the time scales over which the MSC data set was collected, inter-session linear correlation coefficients between scan sessions tended to be quite high across all methods $(0.94-0.99)$. For each subject, inter-session similarity was on average greater for subjects whose BOLD-weighted images were corrected by Synth than when their BOLD-weighted images were corrected using either FSL (FSL-Synth $=4 \mathrm{e}-3 ; \mathrm{p}=0.008 ; \mathrm{t}=-2.84 ; \mathrm{df}=27)$, or ANTs $($ ANTs-Synth $=-0.015 ; \mathrm{p}=1 \mathrm{e}-11$; $\mathrm{t}=-11.25 ; \mathrm{df}=27)$.

We reasoned that improving EPI image consistency would also reduce the variability of the intensities of individual voxels across sessions. To test this, we constructed timeaverage BOLD-weighted images for each session contributed by a subject. We temporally concatenated each of these images, forming a pseudo-time series reflecting session-to-session 
a

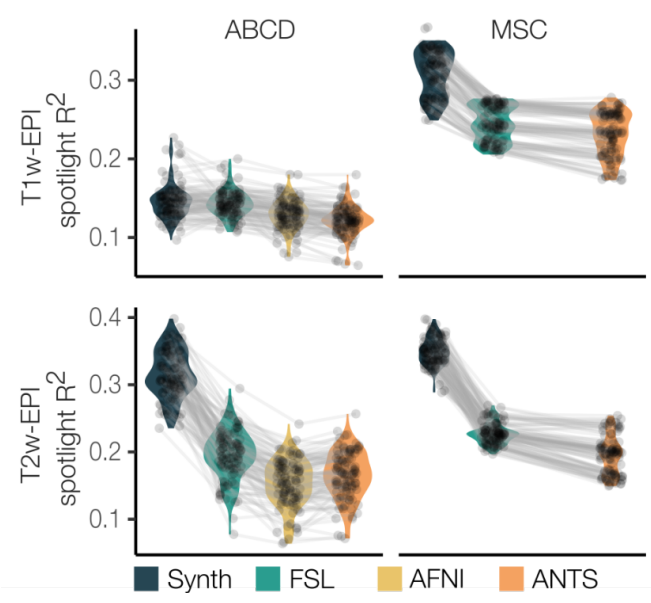

b

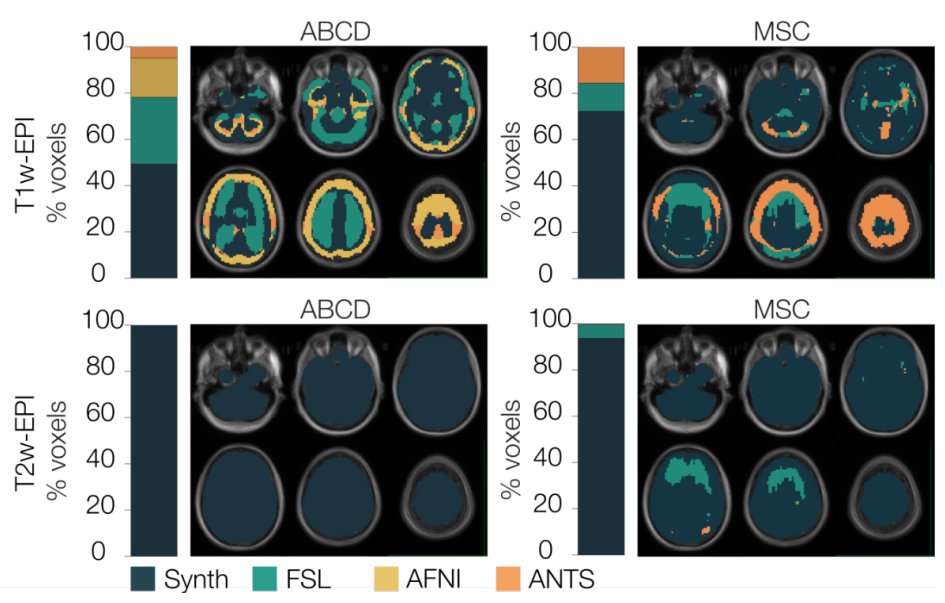

Figure 6: Regional variation in registration performance. Summary of local similarity between the BOLD-weighted images produced by each registration pipeline and their associated $\mathrm{T} 1 \mathrm{w} / \mathrm{T} 2 \mathrm{w}$ anatomical images. (a) Violin plots depicting the distributions of average spotlight $\mathrm{R}^{2}$ value between each subject's BOLD-weighted image and $\mathrm{T} 1 \mathrm{w}$ (top row) and $\mathrm{T} 2 \mathrm{w}$ (bottom row) computed across all gray matter voxels for ABCD (left column) and MSC (right column) data sets. (b) Winner-take-all maps depicting for each brain voxel which pipeline produced the greatest average local correlation between BOLD-weighted image and $\mathrm{T} 1 \mathrm{w} / \mathrm{T} 2 \mathrm{w}$ images. The color of each voxel depicts which registration pipeline produced, on average, the greatest spatial $\mathrm{R}^{2}$ values computed over a centered $7 \times 7 \times 7$ cube. Bars to the left of each set of images depict the percentage of voxels "won" by each registration pipeline.

changes in the BOLD-weighted images. Then, we computed a metric, session-signal-tonoise (s-SNR, analogous to traditional t-SNR), for each voxel defined as the mean intensity of a voxel, $\mu$, divided by the standard deviation of its intensity across sessions, $\sigma$. Lastly, we summarized alignment stability for each subject by averaging s-SNR across all voxels residing within a whole brain mask defined by their subject-specific FreeSurfer parcellation (Figure 7b). Again, we observed that Synth-aligned images exhibited greater session-tosession stability than BOLD-weighted images aligned with FSL (FSL-Synth=-2.58; $\mathrm{p}=3.9 \mathrm{e}-$ $07 ; \mathrm{t}=-6.65 ; \mathrm{df}=27)$ and ANTs (ANTs-Synth $=-3.5 ; \mathrm{p}=1.25 \mathrm{e}-9 ; \mathrm{t}=-9.0 ; \mathrm{df}=27)$.

Next, we explored the possibility that improving the consistency of EPI registration across sessions would also reduce the variability of resting state correlation matrices. To test this, we identically preprocessed (see Supplemental methods) the MSC data sets produced by each registration pipeline and computed the correlation matrices for all gray matter voxels. For each session, we extracted the upper triangle of the associated correlation matrix. Finally, we quantified resting state correlation matrix stability by computing the average pairwise correlation between the upper triangles of each subject's resting state matrices. At the subject level, comparing only the average pairwise correlation matrix similarity (one value per subject and pipeline), we found that correlation matrices from Synth-aligned data sets were significantly less variable than those produced by ANTs (ANTs-Synth=-0.015; $\mathrm{p}=3.16 \mathrm{e}-7 ; \mathrm{t}=-6.7, \mathrm{df}=27)$, but not FSL (FSL-Synth=2.0e-3; $\mathrm{p}=0.39 ; \mathrm{t}=-0.89 ; \mathrm{df}=27) . \quad$ An additional mixed effects analysis of similarity between unique session pairs (that is, not ag- 
gregated into a single subject-level value) revealed BOLD-signal correlation matrices from Synth aligned data sets to be significantly less variable across sessions than those produced by either FSL (FSL-Synth=-2.0e-3; $\mathrm{p}=0.006 ; \mathrm{t}=-2.76 ; \mathrm{df}=1347$ ) or ANTs (ANTs-Synth=0.014; $\mathrm{p}=3.05 \mathrm{e}-81 ; \mathrm{t}=-20.46 ; \mathrm{df}=1347$ ) (Figure $7 \mathrm{c}$ ). We observed a nearly identical pattern of significant results when global signal regression was omitted from the resting state preprocessing (see Supplemental methods).

a

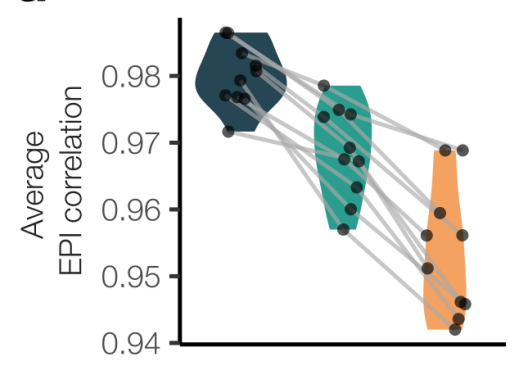

b

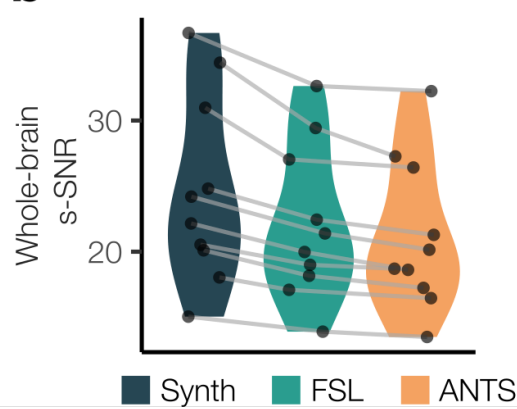

C

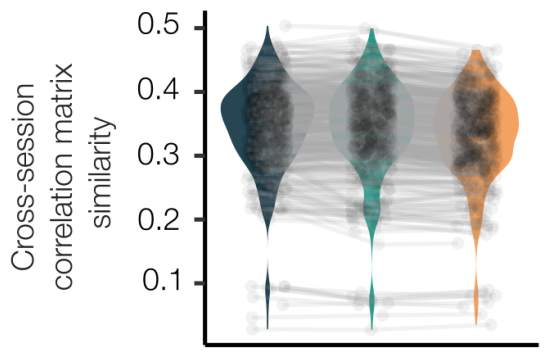

Figure 7: Cross-session reliability metrics. Repeated measurements of the same 10 individuals in the MSC data set allows us to assess the reliability of different approaches to EPI distortion correction. (a) Distributions of the mean pairwise correlation between time-averaged resting state BOLD-weighted volumes. (b) Average voxelwise session-SNR (s-SNR) computed within a whole brain mask. (c) Similarity between the BOLD signal correlation matrices from each unique pair of sessions. Each of the 10 subjects contributed 10 resting state data sets, corresponding to 45 unique session pairs per subject. Similarity is quantified as the correlation between the vectorized upper triangles of the pairs of BOLD signal correlation matrices. Note that these similarity values are smaller than have been reported elsewhere for this same data set as a consequence of the minimal spatial smoothing and the analyses being applied at the voxel as opposed to parcel level.

\subsection{Correction of EPI distortion involves shifting voxels along phase encoding and non- phase-encoding directions.}

Geometric distortion affecting BOLD-weighted images is dominated by voxel shifts in the phase encoding direction, and often only these distortions are corrected. However, other effects produce distortions that are not constrained to the phase encoding direction. These include eddy current distortion; warping due to gradient non-linearities; imperfect slice profiles associated with time-limited RF pulses; chemical shift if fat is not suppressed; the much smaller distortion in the readout direction; and inconsistent phase encode direction due to head pose changes. Errors in affine alignment due to distortion arising from any of these sources are also expected to contribute (see Supplemental Figure 10). Therefore, although we expect distortions in the phase encoding direction to predominate, Synth is not constrained to correct only in the phase encoding direction. Accordingly, we sought to understand the spatial structure of the corrections produced by Synth. We anticipated that the largest component of the distortion-correcting warps would correspond to focal refinements along the phase encoding direction of areas typically associated with susceptibility artifacts (near 
orbitofrontal cortex, ear canals and sinuses), while relatively smaller correction components associated with other sources of distortion would manifest as well.

We created group average EPI correction maps by aligning the nonlinear warps from each subject to the group atlas. Separately for ABCD and MSC data, we computed the mean across subjects for the $\mathrm{x}-, \mathrm{y}^{-}$, and $\mathrm{z}-$ components of the non-linear warps. This allowed us to visualize the distortion corrections that were applied on average to subjects in each data set. While we anticipated that the majority of the EPI distortion-correcting warps would reflect voxels shifting along the phase encoding direction (y-direction; $A B C D=$ posterioranterior; $\mathrm{MSC}=$ anterior-posterior), in both data sets, we observed that warps exhibited complex spatial structure for $\mathrm{x}-, \mathrm{y}-$, and $\mathrm{z}$ - directions, with features closely corresponding to the underlying geometry of the brain and skull (Figure 8). Strong bilateral symmetry was observed in ABCD and MSC warps (Figure 8a, x-displacement), indicating that after affine alignment, the BOLD-weighted images required additional local refinements that involve lateral expansion and contraction that are most prominent along the perimeter of the brain and skull. Affine aligned BOLD-weighted images from both data sets also required local refinements in the phase encoding direction (Figure 8a, y-displacement) that differentially affected orbito-frontal cortex; posterior (occipital and superior parietal) brain/skull boundaries; and the perimeter of the cerebellum. In both data sets, consistent local refinements affecting the superior-inferior placement of the voxels was also required (Figure 8a, z-displacement).

To quantify the degree of post-affine local refinement applied to brain voxels by the distortion-correcting warps, we extracted $\mathrm{x}-, \mathrm{y}$-, and $\mathrm{z}$-displacement values from each component of the group average warps along with the vector norm. In the ABCD data set, the average brain voxel displacement was approximately $1.8 \mathrm{~mm}(\sigma=0.8)$ while displacements in the MSC data set, acquired on an older scanner model (3T Trio), were somewhat larger at $2.65 \mathrm{~mm}(\sigma=1.19 \mathrm{~mm})$. Although we expected the largest component of voxel displacement to lie along the phase encoding direction (y-displacement), sizable displacements were observed in all three directions (Figure 8b,d).

The consistent spatial structure of the warps produced by Synth suggest that sources other than phase encoding distortion contribute significantly to distorting BOLD-weighted images. The presence of non-phase encoding distortion highlights the importance of allowing corrective image deformations to occur in non-phase encoding direction in order to achieve high quality alignment between BOLD-weighted and anatomical images.

\section{Discussion}

Distorted fMRI images are an inevitable consequence of the trade-offs made to achieve a sampling rate sufficient to measure neurally meaningful fMRI signal variability. Complications in registering distorted functional BOLD-weighted images to their undistorted anatomical image counterparts are a perennial challenge faced by neuroimaging researchers. Performed well, proper image registration reduces variability in measurements, improves the statistical power of analyses and produces significant improvements in localization of responses to experimental manipulations [7, 40, 41, 41]. For these reasons, the quality of EPI 


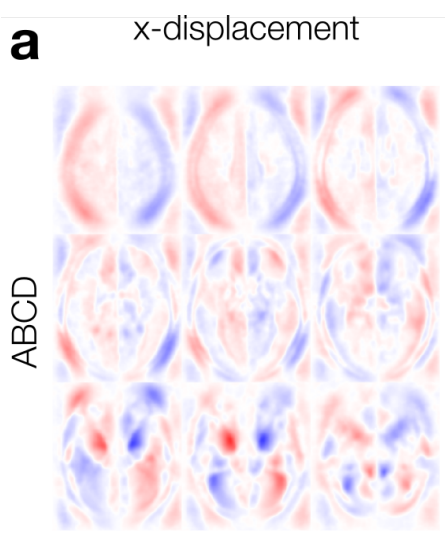

C

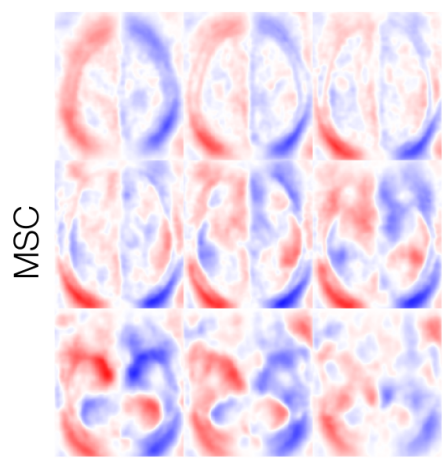

y-displacement phase-encoding direction
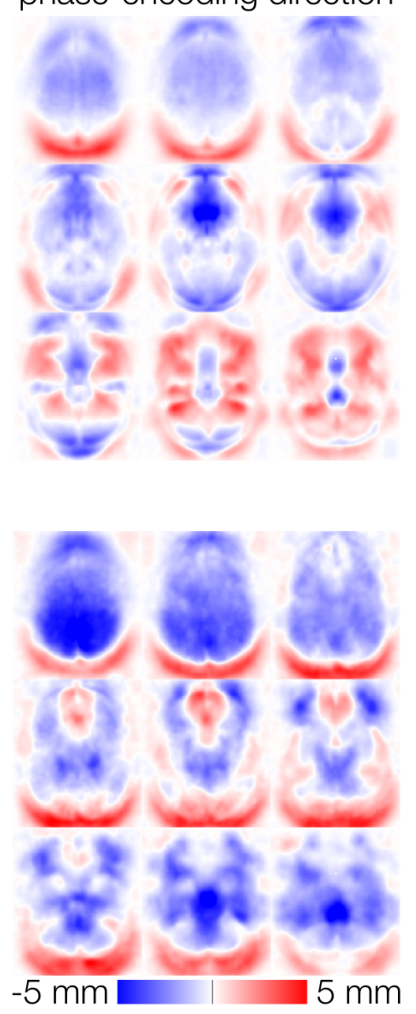

z-displacement
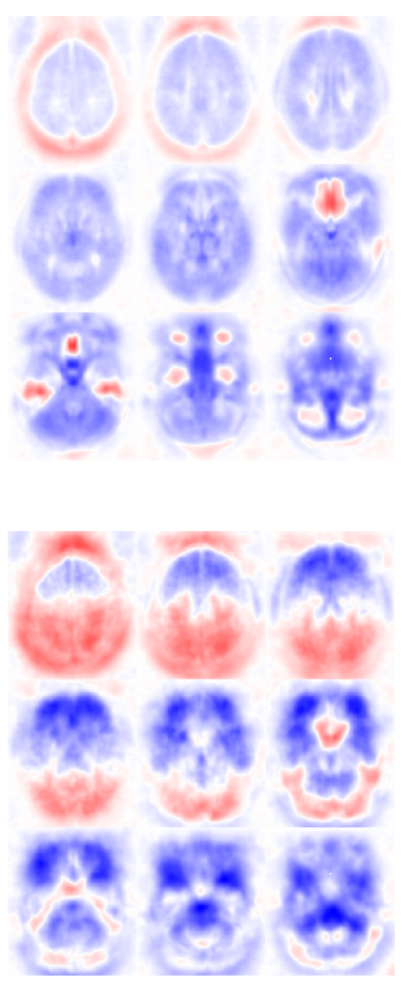

b

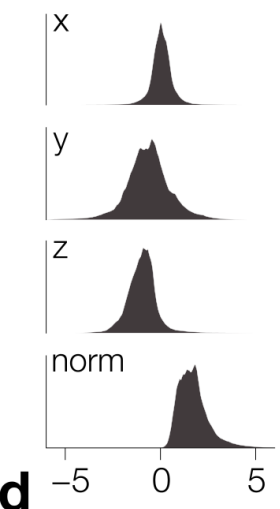

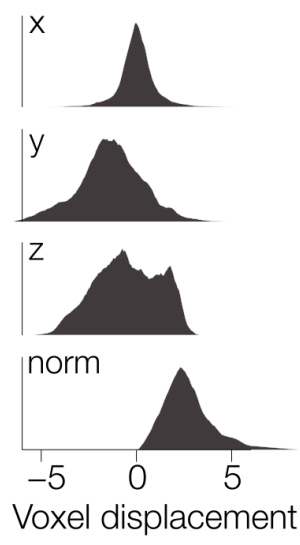

$(\mathrm{mm})$

Figure 8: Group average distortion-correcting warps. (a) Maps of average x-, y-, and z- displacement for voxels in the ABCD data set. (b) Distributions of direction-specific ( $\mathrm{x}-, \mathrm{y}-$, and $\mathrm{z}$-displacement) and displacement magnitude (vector norm) voxel displacement for voxels within a whole brain mask defined by the group atlas. (c) Average displacement maps for the MSC data set. (d) Direction-specific and displacement magnitude distributions for brain voxels in the MSC data set.

distortion correction and anatomical registration is of great concern in every branch of neuroimaging and the primary motivation for developing methods to improve fMRI registration performance.

The most commonly used solution to the EPI distortion problem relies on acquiring additional information about magnetic field inhomogeneity using a separate field map scan $[1,3,4,8]$. Given the expense of acquiring field map data and the complexity of applying it to MRI data, EPI distortion correction methods that do not require additional scan time are desirable. Additionally, older data sets acquired prior to the widespread adoption of field map methods contain uncorrected distortions which make the data unsuitable for modern MRI analysis procedures requiring a high degree of correspondence between functional and anatomical images (e.g., when projecting volumetric data to anatomically derived cortical mesh surfaces). However, correction methods based on directly mapping to undistorted high resolution anatomical images have proven less reliable than methods that explicitly measure 
and correct the EPI distortion [16, 17, 38].

Here, we hypothesized that the reliability of field map-less approaches could be improved if the underlying contrast properties of the BOLD-weighted and anatomical images match more closely. The distortion correction approach that Synth employs is to generate a high resolution synthetic functional image that has nearly the same tissue contrast properties as a BOLD image (95\% similarity) but is based on information from the undistorted geometry of a subjects' T1w and T2w anatomical images. The approach implemented in Synth for constructing a synthetic BOLD-weighted image target can be considered an example of a class of registration procedures termed "mono-modal reduction" [42]. After generating a synthetic target image, a distortion-correcting warp is constructed in the same way as other direct mapping approaches: by directly mapping to an undistorted target image. Here, we have demonstrated that when a synthetic image is used as a target, the resulting fMRI alignment quality rivals and frequently exceeds those produced by standard field map methods.

In typical field map correction procedures, estimates of local distortion are constrained to the phase encoding direction. However, there are many reasons why allowing corrections to occur in non-phase encoding directions may become necessary to achieve correct alignment. For example, due to subject motion occurring between the acquisition of the field map data and the BOLD-weighted data, the phase encoding direction may change from the perspective of a brain-based reference frame, rendering the field map correction suboptimal. Eddy current effects and gradient nonlinearities can also produce image distortion in nonphase encoding directions [43]. Additionally, our analyses have shown that when distortion is present, affine alignment estimates are error-prone. Distortion-induced affine error leaves residual misalignment whose mitigation requires that the correcting warp be free to operate in non-phase encoding directions (see Supplemental Figure 10). A key practical benefit of directly mapping the distorted BOLD-weighted image to an undistorted synthetic image is that these errors can be corrected without explicitly modeling them.

An intriguing potential use case for Synth is to use its warp estimation capabilities to empirically measure these other unspecified components of EPI image distortion. In this regard, we provide a note of caution. While the warps produced by Synth to correct EPI distortions do reflect those areas of the image affected by field inhomogeneities and other true sources of image distortion, they do not reflect these sources solely. Rather, the warps represent a combination of EPI image distortion and errors in the initial affine parameters used to align the BOLD-weighted image to their anatomical counterparts. This occurs because it is difficult, if not impossible, to exactly determine the correct affine parameters that correctly align two images when one of them is distorted. Furthermore, when distortion is present, affine alignment parameters cannot be meaningfully interpreted in anything other than purely operational terms, i.e., the parameters that maximize some image similarity metric. The need to allow for local refinements resulting from incorrect affine registration is the one of the primary motivations for allowing corrections in non-phase encoding directions when aligning to Synth images.

Although the corrections produced by Synth are high quality, several open questions remain relating to how this general approach can be implemented most effectively. Chief 
among these is whether Synth's performance can be augmented further by including existing field map information derived using traditional approaches. The focus of the present report is assessing the quality of the field map corrections produced by Synth in a situation in which there is no initial estimate of the EPI image distortion provided to the underlying nonlinear warping software. Including an existing field map as an initial estimate may increase Synth's performance further. Future iterations of Synth will include this ability which may produce even greater fidelity between functional and anatomical images than what was achieved for the results presented here.

Synth models the difference in effective spatial resolution between T1w/T2w and BOLDweighted images by blurring the radial basis function (RBF) components of the anatomical images with an Epanachnikov smoothing kernel prior to fitting the model to the target BOLD-weighted image. In principle, the optical blurring should occur only after the field map deformation is applied, rather than prior to it. However implementing the model in this way would require repeatedly blurring the high spatial resolution radial basis components of the $\mathrm{T} 1 \mathrm{w} / \mathrm{T} 2 \mathrm{w}$ source images after every iterative update of the estimated field map parameters. A naive implementation of this approach would be computationally prohibitive. Prior research has shown that in practice the sort of approximation implemented in Synth in many cases does not introduce a significant amount of error [44]. However, while the quality of the field map corrections produced by Synth appears to be quite high, some improvement may yet be gleaned by using the "correct model", assuming the technical challenges to its implementation can be surmounted.

That different SynthTarget model specifications were required to achieve similar contrast similarity metrics in the ABCD and MSC data sets suggests that what constitutes a sufficient RBF model for producing high-quality synthetic images is likely to vary from data set to data set. To address this, Synth provides a high degree of flexibility, allowing the user to specify a custom linear model consisting of both main-effect terms and interaction terms between a user-specified number of source images, each decomposable into an user-specified number of RBF components. In general, more complex models will allow Synth to capture more complicated relationships between the voxel intensities of the $\mathrm{T} 1 \mathrm{w} / \mathrm{T} 2 \mathrm{w}$ images and BOLDweighted images. This flexibility comes at the cost of increased memory requirements and computational time, but can produce more accurate synthetic images that may ultimately improve registration quality. In our results, the RBF model parameters were chosen to produce a synthetic image of qualitatively sufficient visual similarity to their associated BOLD-weighted images. By default, Synth uses the RBF model parameters optimized for the ABCD data set (i.e. a 12 component decomposition on the subjects' $\mathrm{T} 1 \mathrm{w} / \mathrm{T} 2 \mathrm{w}$ images, along with pairwise interaction terms). For users looking for guidance on selecting model specifications, our results indicated synthetic images with $95 \%$ Fourier contrast similarity to the target BOLD-weighted image is sufficient for producing high quality corrections. Additional exploration will be required to determine the optimal balance of time and quality.

Even though Synth's underlying RBF model provides a great degree of flexibility in fitting the functions that map T1w/T2w voxel intensities to BOLD-weighted image voxel intensities, other data collection parameters and preprocessing steps may improve the quality of the synthetic images or reduce the needed complexity of the RBF model. For example, ad- 
justing echo times during data acquisition may produce images that can be more accurately modeled with fewer RBF components while still retaining sufficient sensitivity to BOLD contrast. Alternatively or in addition, performing contrast enhancing preprocessing of the anatomical and BOLD-weighted images so that the resulting function relating their voxel intensities can be modeled more easily by the chosen RBF model may allow for simpler RBF models to perform equivalently. In our own preliminary tests and for the results reported here, we observed that synthetic images were more accurate when a bias field was estimated and removed from the anatomical images and image contrast was increased through histogram normalization (see Supplemental methods).

A recent study has revealed that surprisingly large sample sizes - on the order of 2000 or more subjects - are required in order to reliably measure relationships between a variety of neuropsychiatric metrics and resting state BOLD signal correlations [45]. This observation is significant because it suggests the presence of a sizable amount of subject-to-subject variability that remains within fMRI resting state data, even when processed using up to date techniques. It is significant, therefore, that we observed improvement in several metrics quantifying session-to-session reliability. In particular, we observed reduced variability in the structure of individuals' BOLD-weighted images across sessions. Importantly, this reduction in session-to-session structural variability was also reflected in the resulting resting state data sets. We observed significant reduction in session-to-session measurements of BOLD signal correlation structure for data sets corrected with Synth (Figure 7c). These results suggest that high quality correction of image distortion may contribute to reducing overall measurement variability in fMRI research.

The results reported here have demonstrated that it is possible to achieve high-quality EPI distortion correction without field map data. We have shown that field map-less approaches, such as Synth, can perform comparably to existing gold-standard distortion correction approaches, and in fact, surpasses them on many measures of global and local image alignment quality. Removing the reliance on field maps to correct EPI distortion will allow researchers to recover samples with missing or corrupted field maps while maintaining high quality alignment between their anatomical and BOLD-weighted images. Field map-less distortion correction may prove to be a particular asset to researchers studying high motion cohorts, such as pediatric or neuropsychiatric populations, where acquiring high quality field map data is a significant challenge. Reliable field map-less distortion correction shows great promise for negating the complications arising from the acquisition, processing and quality checking of field map data and has the potential to greatly simplify data processing in the neuroimaging field.

\section{Code sharing}

The Synth software package and other utilities and scripts used for this project can be made available upon request and downloaded at https://gitlab.com/vanandrew/omni upon publication. 


\section{Data availability}

The ABCD data repository grows and changes over time. The ABCD data used in this report came from ABCD collection 3165 and the Annual Release 2.0, DOI 10.15154/1503209.

The MSC data set used in this report can downloaded from OpenNeuro.org, DOI 10.18112/openneuro.ds000224.v1.0.3

\section{Acknowledgments}

This work was supported by DA007261 (D.F.M.), MH096773 (D.A.F, N.U.F.D.), MH122066 (D.A.F., N.U.F.D.), MH121276 (D.A.F., N.U.F.D), MH124567 (D.A.F., N.U.F.D.), NS088590 (N.U.F.D.), Kiwanis Neuroscience Research Foundation (N.U.F.D.), the Jacobs Foundation grant 2016121703 (N.U.F.D.).

\section{Competing interests}

E.A.E., D.A.F and N.U.F.D. have a financial interest in NOUS Imaging Inc. and may financially benefit if the company is successful in marketing FIRMM motion monitoring software products. A.N.V., O.M.-D., E.A.E., D.A.F., N.U.F.D. may receive royalty income based on FIRMM technology developed at Oregon Health and Sciences University and Washington University and licensed to NOUS Imaging Inc. D.A.F. and N.U.F.D. are co-founders of NOUS Imaging Inc.

\section{ABCD acknowledgement}

Data used in the preparation of this article were obtained from the Adolescent Brain Cognitive Development (ABCD) Study (https://abcdstudy.org), held in the NIMH Data Archive (NDA). This is a multisite, longitudinal study designed to recruit more than 10,000 children age 9-10 and follow them over 10 years into early adulthood. The ABCD Study is supported by the National Institutes of Health and additional federal partners under award numbers U01DA041022, U01DA041028, U01DA041048, U01DA041089, U01DA041106, U01DA041117, U01DA041120, U01DA041134, U01DA041148, U01DA041156, U01DA041174, U24DA041123, U24DA041147, U01DA041093, and U01DA041025. A full list of supporters is available at https://abcdstudy.org/federal-partners.html. A listing of participating sites and a complete listing of the study investigators can be found at htt ps://abcdstudy.org/scientists/workgroups/. ABCD consortium investigators designed and implemented the study and/or provided data but did not necessarily participate in analysis or writing of this report. This manuscript reflects the views of the authors and may not reflect the opinions or views of the NIH or ABCD consortium investigators. 


\section{Supplemental methods}

\subsection{Description of common pipeline}

Distortion correction methods were compared using a common registration pipeline, which differed only in the distortion correction method and functional-to-T1w alignment. The registration pipeline includes alignment of anatomical images to a common anatomical atlas, functional framewise alignment and resampling of the final aligned functional data to $3 \mathrm{~mm}$. Details of each step are explained in the following sections.

\subsection{Anatomical registration/segmentation}

Anatomical alignment was accomplished with two methods for each data set. For the $\mathrm{ABCD}$ data set, edge images were extracted from each subjects' $\mathrm{T} 1 \mathrm{w}$ and $\mathrm{T} 2 \mathrm{w}$ images and aligned with a rigid body transform using flirt with the correlation ratio cost function. For the MSC data set, we aligned each subjects' $\mathrm{T} 1 \mathrm{w}$ and $\mathrm{T} 2 \mathrm{w}$ images with a rigid body transform using 3dAllineate with a mutual information cost function calculated over $100 \%$ of the voxels. For both methods, the resulting transformation parameters were saved for later use. Both the T1w and T2w images were bias field corrected using N4BiasFieldCorrection [46] ( spline distance $=100$, initial mesh resolution $=1 \times 1 \times 1)$. On the ABCD data set, skullstripping was done on the T1w images with bet [47] (fractional intensity threshold $=0.1$ ). On the MSC data set mri_watershed was used to skullstrip the T1w images. For each subject, we aligned their debiased, skullstripped $\mathrm{T} 1 \mathrm{w}$ volume to a common template, the TRIO_Y_NDC atlas, using a 9-parameter affine transformation estimated with 3dAllineate and a mutual information cost function computed over $100 \%$ of voxels. We then combined the resulting transformations to align both $\mathrm{T} 1 \mathrm{w}$ and $\mathrm{T} 2 \mathrm{w}$ volumes to the TRIO atlas using windowed sinc-function interpolation. Anatomical segmentation was done using recon-all from FreeSurfer 6.0 [48] with the -T2 flag enabled.

\subsection{Alignment of EPI images}

Framewise alignment of EPI images was accomplished using AFNI's 3dAllineate. First, a reference image was constructed by averaging 100 aligned frames from the resting state times series taken from time intervals with the lowest DVARs values [49] [50]. To determine these intervals, a DVARs time series was calculated by computing the temporal derivative of each voxel's time series and normalizing its standard deviation to be equal to 1 . For each frame, a DVARs value was calculated as the spatial mean of the absolute value of the derivative time series. Because we were interested in extracting the frames to construct the reference EPI image from prolonged intervals of relatively low DVARs values, we then low-pass filtered the DVARs time series by bi-directionally filtering it with a 1st order digital Butterworth filter with normalized cut-off frequency of 0.16 . To create the reference image, we selected 100 frames associated with the lowest smoothed DVARs values and aligned them to the individual frame that had the lowest DVARs value in the session using rigid body transformations. Alignment parameters were estimated with 3dAllineate and employed a least-squares cost function along with the -autoweight option. Finally, we constructed a reference image for the session by averaging the 100 aligned frames together. 
Lastly, framewise alignment parameters were estimated by registering each frame individually to the reference image. To reduce the influence of signal drift on framewise registration and to increase the contribution of properly aligned edges to the cost function, we spatially high-pass filtered the reference functional image and each functional frame independently by smoothing them with large $12 \mathrm{~mm}$ FWHM Gaussian kernel and subtracting the result from the original image. These high-pass filtered volumes were then rigid body aligned using 3dAllineate which optimized the least-squares cost function calculated over $75 \%$ of the voxels.

\subsection{Resting state denoising}

Resting state data was denoised by regressing a series of nuisance covariance from each voxel's time series. The design matrix included a constant term; a linear trend component; the first 8 low order Legendre polynomials -to remove low frequency fluctuations; time series corresponding to the 6 framewise rigid-body alignment parameters; and the first derivative of each of the alignment parameters along with two additional lagged copies. In addition, the design matrix included nuisance white matter covariance as well as time series from nearby voxels outside of the brain used to model global and regional nuisance variability [51-54]. The white matter regressors were constructed by extracting and normalizing time series from all voxels labeled as white matter by the subject's FreeSurfer parcellation. Singular value decomposition was then applied to extract 12 time series accounting for the most white matter variance. A similar procedure was used to construct the external signal nuisance regressors. Here, time series from voxels residing within a 5 voxel shell surrounding the brain were extracted. Again, the first 12 singular vectors were computed and included in the denoising design matrix. The complete set of nuisance variables was then projected from the voxel time series using linear regression. We performed this analysis twice, in one instance including the global mean gray matter as an additional nuisance regressor and in the other instance omitting it. Lastly, the regression model residuals were bandpass filtered to retain only frequencies between 0.001 and $0.1 \mathrm{~Hz}$. Frame censoring to remove high motion frames was employed using a weighted correlation approach [50]. First, using the raw fMRI data, we computed a DVARs time series across all brain matter. Frames in which DVARs exceeded two standard deviations were flagged and given a zero weighting. To reduce the influence of any contamination remaining in adjacent frames, the weighting of the three frames preceding and following were also reduced in proportion to their absolute distance from the censored frame. BOLD signal correlation matrices were calculated using these computed weights.

\section{Description of radial basis function model used to estimate the synthetic EPI image}

Synth allows the user to specify a regression model that maps source image intensities to target image intensities. The radial basis components were constructed using the following procedure: Let $\mathbf{I}_{i}$ refer to an individual source image, e.g., T1w or T2w; and $\left\{c_{1}, c_{2}, \ldots c_{n}\right\} \in C$ refers to a set of $n$ evenly spaced values that lie between the maximum and minimum values 
of $\mathbf{I}_{i}$. Then each radial basis component, $\mathbf{r}_{j}$, of an image, $\mathbf{I}_{i}$, is constructed according to the following equation:

$$
\mathbf{r}_{j}\left(\mathbf{I}_{i}\right)=\exp \left(4 \ln (0.5)\left(\frac{\mathbf{I}_{i}-c_{j}}{c_{1}-c_{0}}\right)^{2}\right)
$$

A visual representation of the images produced by applying Equation (3) to $\mathrm{T} 1 \mathrm{w} / \mathrm{T} 2 \mathrm{w}$ images is depicted in Figure 1a. The final design matrix fit by SynthTarget consists of each radial basis component concatenated into a single matrix $\mathbf{F}$, along with intercept terms, $\mathbf{E}_{i}$, for each source image, such that:

$$
\mathbf{F}=\left[\begin{array}{cccccccc}
\mid & \mid & \mid & \mid & \ldots & \mid & \ldots & \mid \\
\mathbf{E}_{0} & \mathbf{E}_{1} & \mathbf{r}_{0}\left(\mathbf{I}_{0}\right) & \mathbf{r}_{1}\left(\mathbf{I}_{0}\right) & \ldots & \mathbf{r}_{0}\left(\mathbf{I}_{1}\right) & \ldots & \mathbf{r}_{j}\left(\mathbf{I}_{i}\right) \\
\mid & \mid & \mid & \mid & \ldots & \mid & \ldots & \mid
\end{array}\right]
$$

For the ABCD data set, $\mathbf{F}$ was constructed from a 12 component decomposition on each of the subjects' $\mathrm{T} 1 \mathrm{w} / \mathrm{T} 2 \mathrm{w}$ images, along with all $\mathrm{T} 1 \mathrm{w} / \mathrm{T} 2 \mathrm{w}$ pairwise interaction terms. As in traditional linear models, these interaction terms are simply the pairwise product of the columns of $\mathbf{F}$. For the MSC data set, a simpler model was sufficient; $\mathbf{F}$ consisted of only a 12 component decomposition of the subjects' $\mathrm{T} 1 \mathrm{w} / \mathrm{T} 2 \mathrm{w}$ images to generate a suitable synthetic BOLD-weighted image. In both ABCD and MSC data sets, $\mathbf{F}$ also included a global intercept term.

\subsection{The blur operator}

While Synth estimates the field map corrections at the $1 \mathrm{~mm}$ isotropic voxel resolution of the $\mathrm{T} 1 \mathrm{w} / \mathrm{T} 2 \mathrm{w}$, the true EPI images are acquired at much lower resolutions. In the case of the MSC data set, this corresponds to a $4 \mathrm{~mm}$ native voxel resolution resampled to 3 $\mathrm{mm}$ isotropic resolution; and for the ABCD data set this corresponds to $2.6 \mathrm{~mm}$ native resolution resampled to $3 \mathrm{~mm}$. When lower resolution EPI images are upsampled to the higher resolution grid, their lower resolution manifests as apparent blur. Synth models this blur (B in Equation (1)) as the convolution of a source image with an Epanachnikov kernel, whose bandwidth is a user specified parameter. This kernel is given by the following expression:

$$
\mathbf{B}(\mathbf{x}, h)=\max \left(0,1-\frac{1}{h}\|\mathbf{x}\|_{2}^{2}\right)
$$

where $\mathbf{x} \in \mathbb{R}^{3}$ is a location on the kernel, and $h$ is the bandwidth parameter. For the results in this paper, the bandwidths of the Epanechnikov kernels were set to $h=4$ and $h=8$ for the ABCD and MSC data set respectively.

\subsection{Contrast correction for error induced image bias}

When source and target images are acquired at substantially different spatial resolutions, or in the early phases of alignment when source and target images are far out of register, 
the synthetic images produced by Synth exhibit significantly "flattened" contrast, even if the rank order voxel intensities comprising the synthetic image largely correspond to those observed in the target image. This is due to the fact that errors in registration or large differences between source and target image resolution bias estimates of the radial basis function parameters, $\boldsymbol{\theta}$, toward the target image mean. In an extreme, but illustrative, case where image registration is so far off that there is no meaningful relationship between corresponding source and target image voxels, the synthetic image will simply be a volume in which all voxels have the same intensity as the target image mean. That is, it will have perfectly 'flat' contrast. As registration improves some, the synthetic image will begin to exhibit more accurately the contrast properties of the target image. In general, however, the contrast of naively estimated synthetic images will tend to be somewhat flattened due to error. To address this, Synth includes a tone curve adjustment option that increases contrast of the final synthetic image. This is accomplished by passing the synthetic image voxel intensities through a monotonic non-linear curve, a process similar to tone curve adjustment in traditional image processing. The curve is constrained to reside within the set of beta function cumulative distributions -distributions well suited for modeling tone curves that tend to be sigmoidal- and the parameters are optimized by minimizing the negative linear correlation coefficient between the target and synthetic images.

\subsection{Estimating field maps with an undistorted synthetic BOLD-weighted image}

The warp parameters that map the undistorted synthetic BOLD-weighted image to the reference BOLD-weighted image were estimated using a three step iterative procedure. The final iteration of this procedure produced the estimated field map corrections used for our analyses. This procedure was conducted as follows:

1. Using SynthTarget, construct an initial synthetic BOLD-weighted image based on the current best affine alignment between $\mathrm{T} 1 \mathrm{w}, \mathrm{T} 2 \mathrm{w}$ and reference BOLD-weighted images (see Synth Target joint optimization). An EPI signal-to-noise mask was used to reduce the contribution of low signal-to-noise areas when constructing the synthetic BOLDweighted image (see Creating the EPI signal-to-noise mask).

2. Estimate a warp that maps the current synthetic BOLD-weighted image to the reference BOLD-weighted image. With the exception of the first iteration, each warp estimate is initialized from the warp estimate of the previous iteration.

3. Apply the inverse of this warp, which approximates a field map correction, to the reference BOLD-weighted image.

4. Repeat steps 1-3, at each iteration, using SynthTarget to re-estimate the synthetic BOLD-weighted image using the most recently corrected BOLD-weighted image (see Equation 1).

For the results presented here, we estimated the field maps using the ANTs SyN algorithm. SyN parameters were set to use a neighborhood cross-correlation cost function (radius $=2$ ), update and total field variance parameters of 0 , winsorize limits of [0.005, 0.995], histogram matching, BSpline interpolation of order 5, convergence parameters of 100x50x50, $1 \mathrm{e}-6$ and 
15, smoothing parameters of $2 \times 1 \times 0$, and shrink factor parameters $4 \times 3 \times 2$. ANTs SyN was run for 3 iterations and each subsequent iteration used a smaller gradient step size of 3,1 , and 0.1 respectively. In order to leverage the detailed spatial information available in the high resolution synthetic BOLD-weighted images when estimating the field map, the warps at each iteration were constructed in the $1 \mathrm{~mm} \times 1 \mathrm{~mm} \times 1 \mathrm{~mm}$ native resolution of the $\mathrm{T} 1 \mathrm{w} / \mathrm{T} 2 \mathrm{w}$ source images. Because EPI distortion affects the accuracy of affine registration, field map solutions were not constrained to the phase encoding direction only.

\subsection{Creating the EPI signal-to-noise mask}

To construct a binary mask that would serve to label EPI voxels suffering from a high degree of noise or signal dropout we implemented a linear discriminant analysis procedure. We began by using the brain mask of the T1w image and aligned it to the BOLD-weighted data set using the affine transformation generated from SynthTarget. This mask served as an initial guess, or prior estimate, for which voxels were likely to represent meaningful fMRI signals in the resting state data set, and those which represent noise and areas of signal dropout (Supplemental Figure 9). Using this initial labeling, we implemented a betweenclass linear discriminant analysis (LDA) based, first, on the raw un-centered and un-scaled voxel time series and then computed the projection of each voxel's time series onto the resulting principal discriminant vector. We repeated this LDA procedure on the centered and normalized time series from each voxel, again projecting the times series back onto the principal discriminant vector. Thus, each voxel was associated with two projection values. We then created a third value associated with each voxel by computing the product of the two principal discriminant vector projections. A second level of LDA was done on these three values. Lastly, Otsu's method [55] was used to label each voxel as 'signal' or 'noise', depending upon its projection onto this final 3-element principal discriminant vector. We refined this labeling by repeating the entire LDA procedure over 20 iterations. During each subsequent iteration the labels produced by the previous iteration were used initialize the current LDA procedure. Sufficiently iterated, this approach converges to a stable binary signal-or-noise label for each voxel. Lastly, to construct a mask containing only within-brain noise voxels — voxels most likely to be affected by signal dropout - we masked the final result of this iterative process to remove non-brain voxels (defined by the skullstrip mask) and morphologically open and dilate the image by 1 voxel. A smoothed version of this mask was used as a weighting volume to further refine the estimate of the synthetic image by reducing the contributions of brain regions where no correspondence could exist between BOLD-weighted images and $\mathrm{T} 1 \mathrm{w} / \mathrm{T} 2 \mathrm{w}$ images due to signal drop out. This procedure is implemented as a standalone function called synthnoisemask as part of Synth software package.

\subsection{SynthTarget joint optimization}

Our goal is to find the optimal set of affine parameters, $\star \gamma$, and radial basis weights, $\star \boldsymbol{\theta}$, that best satisfies the model described by Equation (1). By applying a maximum likelihood estimator, we obtain the following expression:

$$
\{\star \boldsymbol{\gamma}, \star \boldsymbol{\theta}\}=\underset{\boldsymbol{\gamma}, \boldsymbol{\theta}}{\arg \min }\left\|\mathbf{y}-\mathbf{M}_{\boldsymbol{\gamma}} \mathbf{B F} \boldsymbol{\theta}\right\|_{2}^{2}
$$



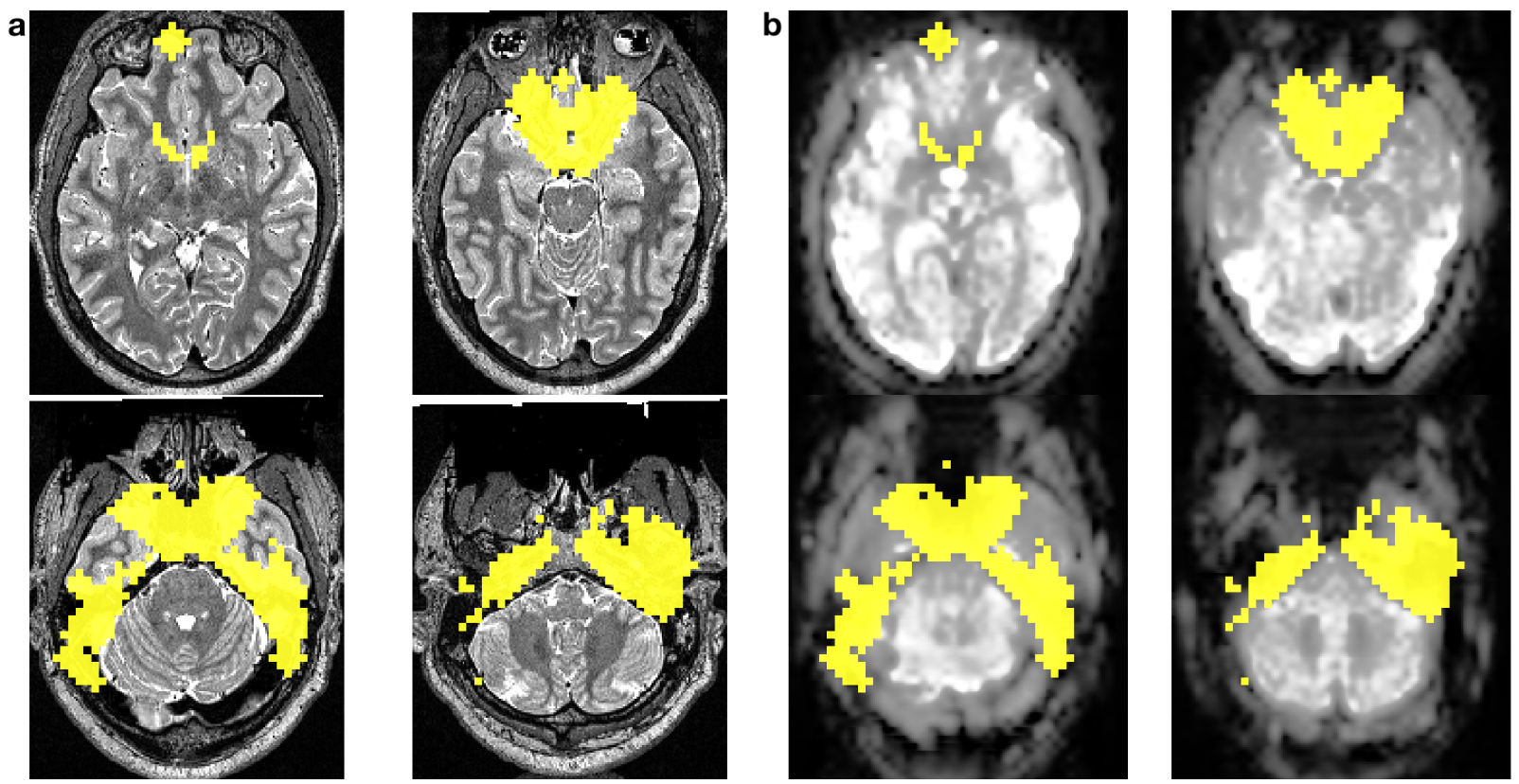

Figure 9: Representative signal-to-noise exclusion mask. (a) Example subject T2w images (underlay) depicting slices that typically contain regions of significant signal loss in EPI scans. (b) The corresponding closely aligned slices from the subject's BOLD-weighted data. In both sets of images, the yellow overlay depicts regions of extreme signal loss detected with the LDA procedure outlined above. In these regions of extreme signal loss, no correspondence exists between a subjects' anatomical and BOLD-weighted data. Therefore, when constructing the synthetic image, the contributions of voxels in this region are downweighted when running SynthTarget. Note qualitative similarity between the noise mask detected with this method and group average map of fMRI signal loss reported in [56].

which is the least squares solution to Equation (1). In practice, Equation (6) is weighted by the EPI signal-to-noise mask to downweight areas of signal dropout in the image.

To find both sets of optimal parameters, we employ an alternating minimization approach [57], which solves for both $\star \boldsymbol{\gamma}$ and $\star \boldsymbol{\theta}$ by fixing one set of parameters to estimate the other over a number of iterations, $N$. First, $\gamma$ is initialized to some initial estimate, $\gamma_{0}$. In this approach, the alternating minimization algorithm is sensitive to the choice of $\gamma_{0}$, as convergence is only guaranteed when the support of the function satisfies the marginal convexity properties described in Theorem 4.3 in [57]. We attempt to satisfy this constraint by using the rigid body alignment solution from $3 d$ Allineate as the initial estimate of $\gamma_{0}$. To find $\boldsymbol{\theta}_{n}$, we fix the value of $\boldsymbol{\gamma}_{n}$ at its current estimate and solve for $\boldsymbol{\theta}_{n}$ using the following expression:

$$
\boldsymbol{\theta}_{n}=\left(\mathbf{F}^{T} \mathbf{B}^{T} \mathbf{B F}\right)^{-1} \mathbf{F}^{T} \mathbf{B}^{T} \mathbf{M}_{\boldsymbol{\gamma}}^{-1} \mathbf{y}
$$

which is the least squares solution to the system $\mathbf{M}_{\boldsymbol{\gamma}}^{-1} \mathbf{y}=\mathbf{B F} \boldsymbol{\theta}$. Once $\boldsymbol{\theta}_{n}$ has been found using the current estimate of affine parameters, a new estimate, $\boldsymbol{\gamma}_{n+1}$, is found by fixing $\boldsymbol{\theta}_{n}$ and generating a gradient descent step that solves Equation 6. A closed form solution to 
the gradient can be found by applying the chain rule [58]:

$$
\mathbf{g}_{\mathbf{n}}=\left(\frac{\partial \mathbf{x}}{\partial \boldsymbol{\gamma}}\right)^{T}\left(\frac{\partial \mathbf{M}_{\boldsymbol{\gamma}} \mathbf{F} \boldsymbol{\theta}}{\partial \mathbf{x}}\right)^{T}\left(\frac{\partial \mathbf{J}}{\partial \mathbf{M}_{\boldsymbol{\gamma}} \mathbf{F} \boldsymbol{\theta}}\right)=\mathbf{P}^{T} \mathbf{G}^{T}\left(\mathbf{M}_{\boldsymbol{\gamma}} \mathbf{B F} \boldsymbol{\theta}-\mathbf{y}\right)
$$

where, $\mathbf{J}$ is the functional expression defined in Equation (6), G is a matrix containing the spatial gradient of the target image, and $\mathbf{P}$ is a padded matrix containing the position of each voxel in the source image. Here, we use the terms "target" and "source" to refer to the synthetic image obtained after applying an affine transformation (i.e., $\mathbf{M}_{\boldsymbol{\gamma}} \mathbf{B F} \boldsymbol{\theta}$ ) and the untransformed synthetic image (i.e., $\mathbf{B F} \boldsymbol{\theta}$ ) respectively. A construction of the $\mathbf{G}$ and $\mathbf{P}$ terms is described below:

$$
\begin{aligned}
& \mathbf{G}=\left[\operatorname{diag}\left(\mathbf{G}_{\mathbf{x}}\right) \operatorname{diag}\left(\mathbf{G}_{\mathbf{y}}\right) \quad \operatorname{diag}\left(\mathbf{G}_{\mathbf{z}}\right)\right] \\
& \mathbf{P}=\left[\begin{array}{llllllllllllll}
\mathbf{x} & \mathrm{y} & \mathrm{z} & \mathrm{e} & & & & & & & & \\
& & & & \mathrm{x} & \mathbf{y} & \mathrm{z} & \mathrm{e} & & & & \\
& & & & & & & & \mathrm{x} & \mathrm{y} & \mathrm{z} & \mathrm{e}
\end{array}\right]
\end{aligned}
$$

where $\mathbf{G}_{x}, \mathbf{G}_{y}$, and $\mathbf{G}_{z}$ are each components of the target image gradient of $\boldsymbol{x}, \boldsymbol{y}$, and $\boldsymbol{z}$, which denote the physical location of each voxel (in scanner space) along each axis in the source image, and $\mathbf{e}$ is defined to be a vector of all ones. To avoid optimizing to saddle points over the non-convex objective function of Equation (6), we use a Nesterov Adam update for each gradient descent iteration with parameter settings $\eta=10^{-3}, \epsilon=10^{-8}, \beta_{1}=0.9$, and $\beta_{2}=0.999$ [59]. Algorithm 1 describes the full joint optimization procedure:

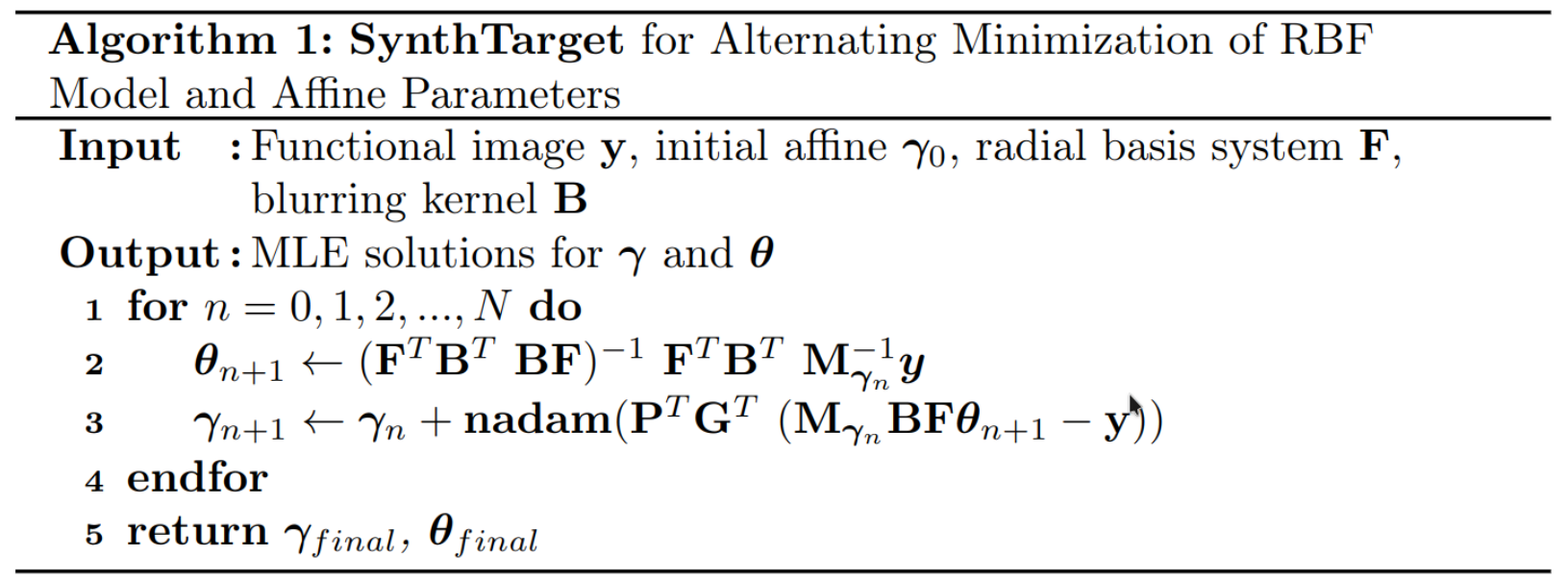

During estimation of $\boldsymbol{\gamma}$ and $\boldsymbol{\theta}$, Algorithm 1 is used in a Gaussian resolution pyramid, where estimated parameters are calculated first on downsampled versions of the input source and target images and subsequently refined on a higher resolution set of images. This process 
increases the speed of the registration procedure by reducing the number of voxels that the cost function is computed over, in addition to aiding in the avoidance of local minima that correspond to locally optimal alignment solutions that are nevertheless wrong. For the results presented here, the Synth joint optimization is repeated for image scales of $4 \mathrm{~mm}, 2$ $\mathrm{mm}$, and $1 \mathrm{~mm}$. Initialization of the affine parameters, $\gamma_{0}$ is estimated by using an external program (i.e., AFNI 3dAllineate) to coregister the target EPI to the anatomical T2 with a mutual information cost function.

\subsection{Distortion correction using alternating descent optimization with SynthUnwarp}

Once the affine parameters are found by SynthTarget, we run a second alternating minimization step that jointly solves for the radial basis function parameters, $\boldsymbol{\theta}$ as well as the non-linear deformation parameters, $\boldsymbol{\phi}$ described in Equation (2), while keeping the affine parameters fixed. An initial warp is estimated by non-linearly aligning the BOLD-weighted image to the $\mathrm{T} 2 \mathrm{w}$ image. This initial warp estimate, $\phi_{0}$, is used as a coarse estimate of the EPI distortion, allowing for a more accurate estimate synthetic BOLD-weighted image. Algorithm 2 summarizes the full distortion correction algorithm:

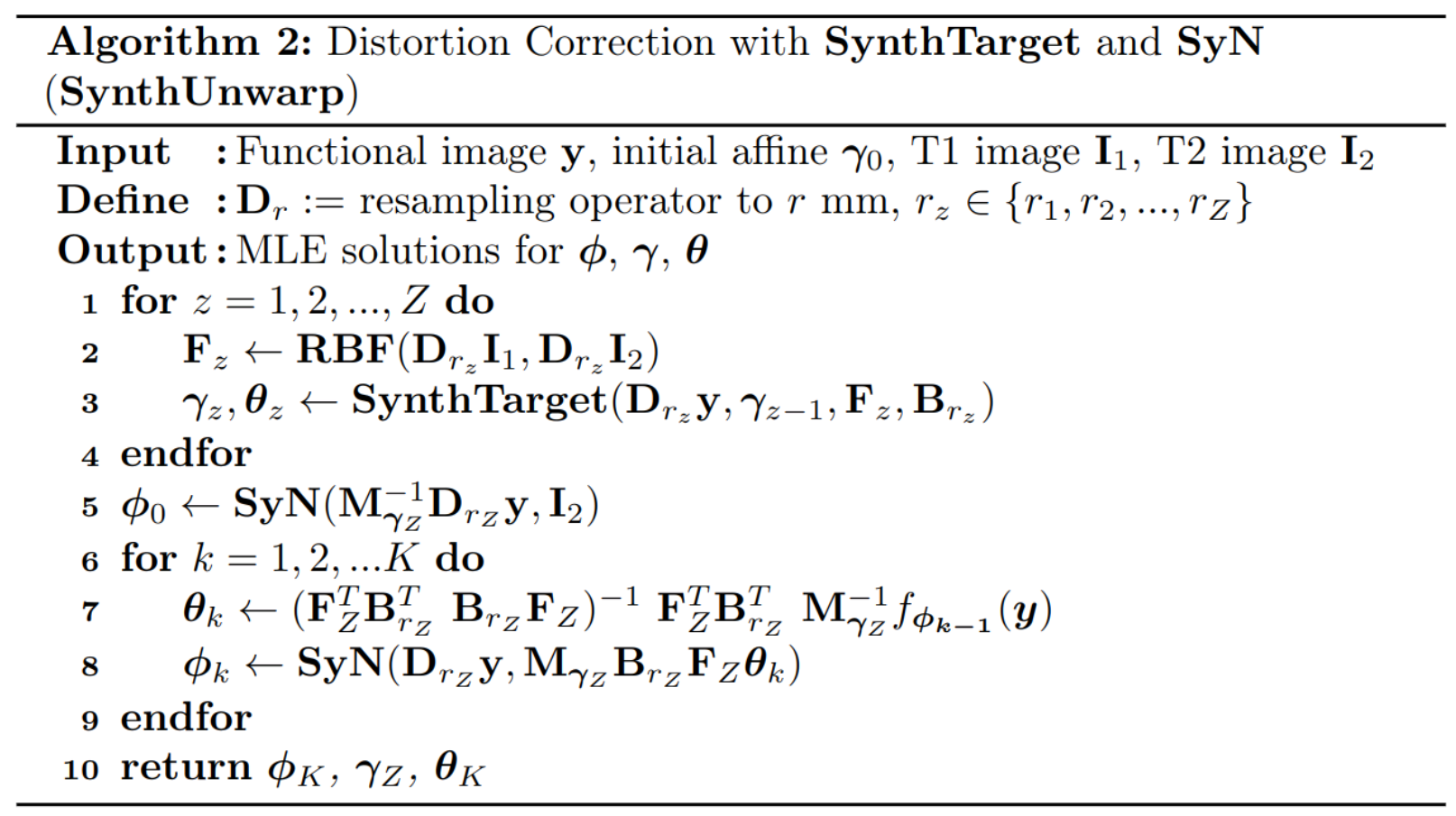

For the results presented here, ANTs SyN was used to find the deformation parameters. Typically, field map correction procedures allow only for geometric deformations to occur in the phase encoding direction. However, in a set of ancillary analyses we observed that the presence of phase-encoding-direction-only geometric distortion introduces significant errors in affine alignment parameters (Supplemental Figure 10). In order to correct the distortion 
bioRxiv preprint doi: https://doi.org/10.1101/2021.03.13.435270; this version posted March 15, 2021. The copyright holder for this preprint (which was not certified by peer review) is the author/funder. All rights reserved. No reuse allowed without permission.

induced affine alignment errors, we elected to allow SyN to estimate distortion corrections that were not constrained to lie along the phase-encoding direction. 


\section{Supplemental material}

13.1. Table of scanner manufacturers and models comprising the ABCD data set

Table 1: Distribution of ABCD data sets acquired by specific scanner models

\begin{tabular}{lll}
\hline Manufacturer & Model & Count \\
GE MEDICAL SYSTEMS & DISCOVERY MR750 & 18 \\
GE MEDICAL SYSTEMS & SIGNA Creator & 1 \\
Philips Medical Systems & Achieva dStream & 1 \\
Philips Medical Systems & Ingenia & 6 \\
SIEMENS & Prisma & 33 \\
SIEMENS & Prisma Fit & 41 \\
\hline
\end{tabular}




\subsection{Statistical tables for global similarity metrics ( $A B C D$ data set)}

AUC ventricles/adjacent white matter voxels

\begin{tabular}{lrrrrrrr}
\hline Name & Estimate & SE & tStat & DF & pValue & Lower & Upper \\
\hline SYNTH (Intercept) & 0.6868 & 0.0092 & 74.3 & 396 & $1.3 \mathrm{e}-234$ & 0.6686 & 0.705 \\
FSL & 0.0226 & 0.0055 & 4.1 & 396 & $5.7 \mathrm{e}-05$ & 0.0117 & 0.033 \\
AFNI & 0.0079 & 0.0055 & 1.4 & 396 & $1.6 \mathrm{e}-01$ & -0.0030 & 0.019 \\
SyN SDC & 0.0090 & 0.0055 & 1.6 & 396 & $1.1 \mathrm{e}-01$ & -0.0019 & 0.020 \\
\hline
\end{tabular}

AUC adjacent interior/exterior voxels

\begin{tabular}{lrrrrrrr}
\hline Name & Estimate & SE & tStat & DF & pValue & Lower & Upper \\
\hline SYNTH (Intercept) & 0.8160 & 0.0043 & 188.4 & 396 & $0.0 \mathrm{e}+00$ & 0.807 & 0.8245 \\
FSL & -0.0133 & 0.0021 & -6.2 & 396 & $1.5 \mathrm{e}-09$ & -0.018 & -0.0091 \\
AFNI & -0.0095 & 0.0021 & -4.4 & 396 & $1.2 \mathrm{e}-05$ & -0.014 & -0.0053 \\
SyN SDC & -0.0311 & 0.0021 & -14.5 & 396 & $2.4 \mathrm{e}-38$ & -0.035 & -0.0268 \\
\hline
\end{tabular}

AUC adjacent gray/white matter voxels

\begin{tabular}{lrrrrrrr}
\hline Name & Estimate & SE & tStat & DF & pValue & Lower & Upper \\
\hline SYNTH (Intercept) & 0.648 & 0.0038 & 172 & 396 & $0.0 \mathrm{e}+00$ & 0.640 & 0.655 \\
FSL & -0.032 & 0.0024 & -13 & 396 & $1.2 \mathrm{e}-33$ & -0.037 & -0.027 \\
AFNI & -0.063 & 0.0024 & -26 & 396 & $8.8 \mathrm{e}-88$ & -0.068 & -0.058 \\
SyN SDC & -0.036 & 0.0024 & -15 & 396 & $1.1 \mathrm{e}-39$ & -0.040 & -0.031 \\
\hline
\end{tabular}

T2 - EPI grad. norm. correlation

\begin{tabular}{lrrrrrrr}
\hline Name & Estimate & SE & tStat & DF & pValue & Lower & Upper \\
\hline SYNTH (Intercept) & 0.62 & 0.0073 & 86 & 396 & $1.1 \mathrm{e}-257$ & 0.61 & 0.64 \\
FSL & -0.11 & 0.0049 & -23 & 396 & $2.3 \mathrm{e}-75$ & -0.12 & -0.10 \\
AFNI & -0.17 & 0.0049 & -34 & 396 & $2.1 \mathrm{e}-119$ & -0.18 & -0.16 \\
SyN SDC & -0.13 & 0.0049 & -26 & 396 & $5.4 \mathrm{e}-87$ & -0.14 & -0.12 \\
\hline
\end{tabular}


T1 - EPI grad. norm. correlation

\begin{tabular}{lrrrrrrr}
\hline Name & Estimate & SE & tStat & DF & pValue & Lower & Upper \\
\hline SYNTH (Intercept) & 0.286 & 0.0076 & 37.6 & 396 & $1.4 \mathrm{e}-132$ & 0.271 & 0.3012 \\
FSL & -0.012 & 0.0039 & -3.0 & 396 & $2.8 \mathrm{e}-03$ & -0.020 & -0.0041 \\
AFNI & -0.037 & 0.0039 & -9.5 & 396 & $2.3 \mathrm{e}-19$ & -0.045 & -0.0295 \\
SyN SDC & -0.018 & 0.0039 & -4.5 & 396 & $8.5 \mathrm{e}-06$ & -0.025 & -0.0100 \\
\hline
\end{tabular}

T2 - EPI normalized MI

\begin{tabular}{lrrrrrrr}
\hline Name & Estimate & SE & tStat & DF & pValue & Lower & Upper \\
\hline SYNTH (Intercept) & 0.154 & 0.00060 & 257 & 396 & $0.0 \mathrm{e}+00$ & 0.153 & 0.155 \\
FSL & -0.013 & 0.00049 & -27 & 396 & $8.8 \mathrm{e}-93$ & -0.014 & -0.012 \\
AFNI & -0.017 & 0.00049 & -35 & 396 & $1.4 \mathrm{e}-122$ & -0.018 & -0.016 \\
SyN SDC & -0.017 & 0.00049 & -36 & 396 & $1.1 \mathrm{e}-126$ & -0.018 & -0.017 \\
\hline
\end{tabular}

T1 - EPI normalized MI

\begin{tabular}{lrrrrrrr}
\hline Name & Estimate & SE & tStat & DF & pValue & Lower & Upper \\
\hline SYNTH (Intercept) & 0.1342 & 0.00044 & 303 & 396 & $0.0 e+00$ & 0.1333 & 0.1351 \\
FSL & -0.0039 & 0.00030 & -13 & 396 & $5.8 e-32$ & -0.0045 & -0.0033 \\
AFNI & -0.0062 & 0.00030 & -21 & 396 & $2.2 e-64$ & -0.0068 & -0.0056 \\
SyN SDC & -0.0072 & 0.00030 & -24 & 396 & $3.3 e-79$ & -0.0078 & -0.0066 \\
\hline
\end{tabular}




\subsection{Statistical tables for global similarity metrics (MSC data set)}

AUC ventricles/adjacent white matter voxels

\begin{tabular}{lrrrrrrr}
\hline Name & Estimate & SE & tStat & DF & pValue & Lower & Upper \\
\hline SYNTH (Intercept) & 0.864 & 0.0156 & 55 & 297 & $9.1 \mathrm{e}-159$ & 0.834 & 0.895 \\
FSL & -0.047 & 0.0029 & -16 & 297 & $1.1 \mathrm{e}-42$ & -0.052 & -0.041 \\
SyN SDC & -0.091 & 0.0029 & -32 & 297 & $1.2 \mathrm{e}-97$ & -0.097 & -0.086 \\
\hline
\end{tabular}

AUC adjacent interior/exterior voxels

\begin{tabular}{lrrrrrrr}
\hline Name & Estimate & SE & tStat & DF & pValue & Lower & Upper \\
\hline SYNTH (Intercept) & 0.811 & 0.0101 & 80 & 297 & $1.1 \mathrm{e}-203$ & 0.791 & 0.831 \\
FSL & -0.025 & 0.0012 & -21 & 297 & $4.5 \mathrm{e}-62$ & -0.027 & -0.023 \\
SyN SDC & -0.021 & 0.0012 & -18 & 297 & $2.3 \mathrm{e}-49$ & -0.023 & -0.019 \\
\hline
\end{tabular}

AUC adjacent gray/white matter voxels

\begin{tabular}{lrrrrrrr}
\hline Name & Estimate & SE & tStat & DF & pValue & Lower & Upper \\
\hline SYNTH (Intercept) & 0.683 & 0.0075 & 91 & 297 & $7.4 \mathrm{e}-219$ & 0.668 & 0.698 \\
FSL & -0.080 & 0.0016 & -49 & 297 & $3.2 \mathrm{e}-144$ & -0.083 & -0.077 \\
SyN SDC & -0.088 & 0.0016 & -54 & 297 & $2.0 \mathrm{e}-155$ & -0.091 & -0.085 \\
\hline
\end{tabular}

T2 - EPI grad. norm. correlation

\begin{tabular}{lrrrrrrr}
\hline Name & Estimate & SE & tStat & DF & pValue & Lower & Upper \\
\hline SYNTH (Intercept) & 0.62 & 0.0088 & 71 & 297 & $4.0 \mathrm{e}-188$ & 0.61 & 0.64 \\
FSL & -0.12 & 0.0021 & -54 & 297 & $1.1 \mathrm{e}-156$ & -0.12 & -0.11 \\
SyN SDC & -0.15 & 0.0021 & -69 & 297 & $2.6 \mathrm{e}-185$ & -0.15 & -0.14 \\
\hline
\end{tabular}


T1 - EPI grad. norm. correlation

\begin{tabular}{lrrrrrrr}
\hline Name & Estimate & SE & tStat & DF & pValue & Lower & Upper \\
\hline SYNTH (Intercept) & 0.505 & 0.0147 & 34 & 297 & $2.5 \mathrm{e}-105$ & 0.476 & 0.534 \\
FSL & -0.103 & 0.0018 & -58 & 297 & $3.9 \mathrm{e}-163$ & -0.106 & -0.099 \\
SyN SDC & -0.095 & 0.0018 & -53 & 297 & $3.8 \mathrm{e}-154$ & -0.099 & -0.092 \\
\hline
\end{tabular}

T2 - EPI normalized MI

\begin{tabular}{lrrrrrrr}
\hline Name & Estimate & SE & tStat & DF & pValue & Lower & Upper \\
\hline SYNTH (Intercept) & 0.157 & 0.00095 & 165 & 297 & $2.3 e-294$ & 0.156 & 0.159 \\
FSL & -0.014 & 0.00024 & -58 & 297 & $6.9 e-164$ & -0.014 & -0.013 \\
SyN SDC & -0.019 & 0.00024 & -82 & 297 & $1.7 e-205$ & -0.020 & -0.019 \\
\hline
\end{tabular}

T1 - EPI normalized MI

\begin{tabular}{lrrrrrrr}
\hline Name & Estimate & SE & tStat & DF & pValue & Lower & Upper \\
\hline SYNTH (Intercept) & 0.152 & 0.00115 & 131 & 297 & $3.7 \mathrm{e}-265$ & 0.149 & 0.154 \\
FSL & -0.013 & 0.00026 & -49 & 297 & $2.6 \mathrm{e}-145$ & -0.013 & -0.012 \\
SyN SDC & -0.016 & 0.00026 & -64 & 297 & $1.8 \mathrm{e}-175$ & -0.017 & -0.016 \\
\hline
\end{tabular}




\subsection{Distortion affecting only the phase encoding direction reduces affine alignment accuracy}

In order to quantify the effect of EPI distortion on affine alignment performance, we applied known rigid body transformations to synthetic BOLD-weighted images that were either distorted or undistorted and then assessed the ability of an affine registration algorithm (MATLAB, imregtform) to correctly realign them to the original undistorted image. EPI distortions were generated by applying only the phase encoding direction component of distortion correcting warps generated by SynthUnwarp. We applied this procedure to one session of data from each of the ten MSC subjects. We separately applied transformations, $\mathbf{R}$, corresponding to pure translations $(\mp 5 \mathrm{~mm}$ ) and rotations ( $\mp 5$ degrees) along or about the three cardinal axes, to the distorted and undistorted synthetic BOLD-weighted images. Next, we estimated $\mathbf{R}^{\mathbf{- 1}}$ for the distorted and undistorted images, by aligning them to the original undistorted image as a target. If the alignment algorithm correctly estimates $\mathbf{R}^{-1}$, then the product $\mathbf{R R}^{-1}$ should be equal to the identity matrix, which has a 2-norm of 1 . Errors in affine alignment will result in deviations from identity in the $\mathbf{R R}^{\mathbf{- 1}}$ product. We therefore quantified the alignment error as the 2-norm of $\mathbf{R R}^{-1}$.

We observed that when no phase encoding distortion was present, imregtform reliably estimated the correct alignment parameters, resulting in $\mathbf{R R}^{-1}$ with a 2-norm that was almost always equal to 1 (Figure 10 left). However, when distortion was present, alignment parameters exhibited greater error (Figure 10 right). 


\section{Undistorted}

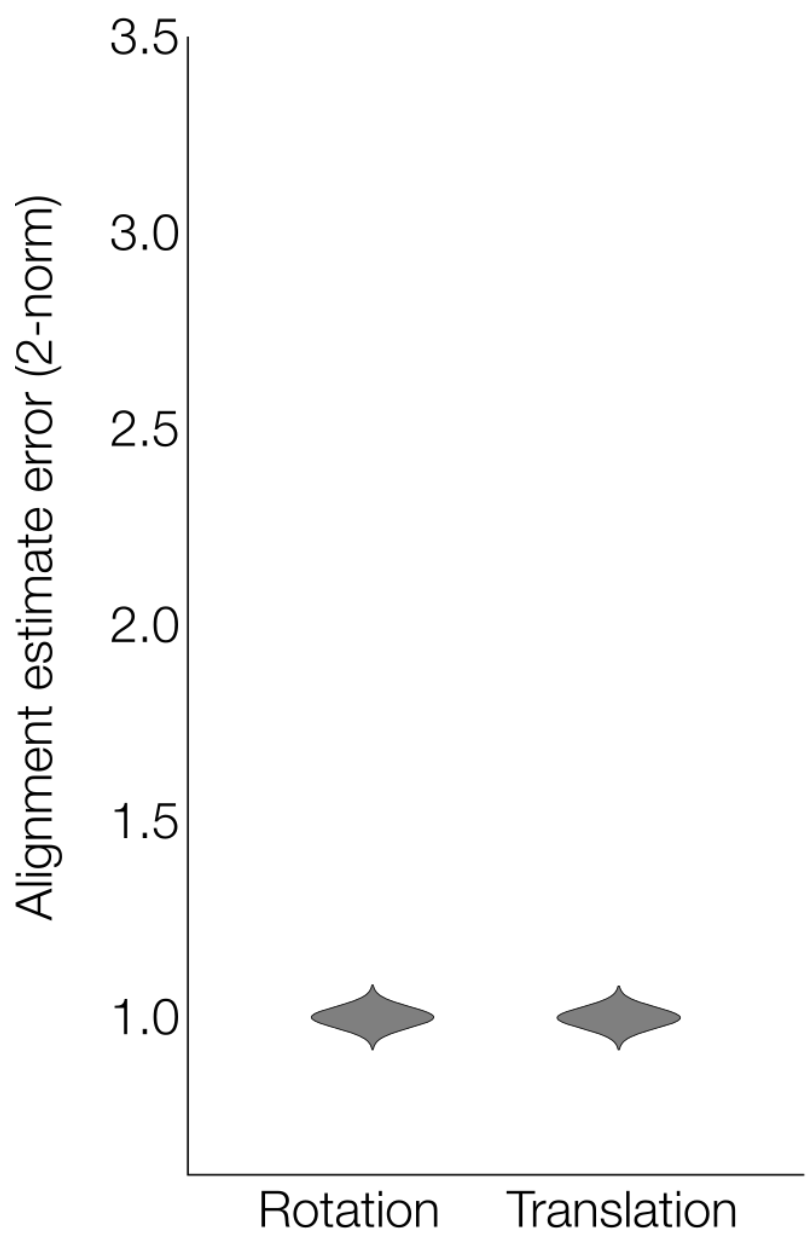

\section{Undistorted}

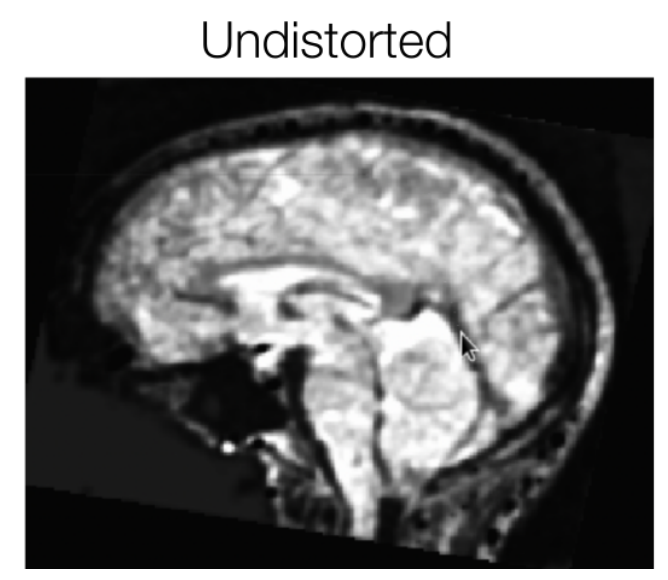

Distorted
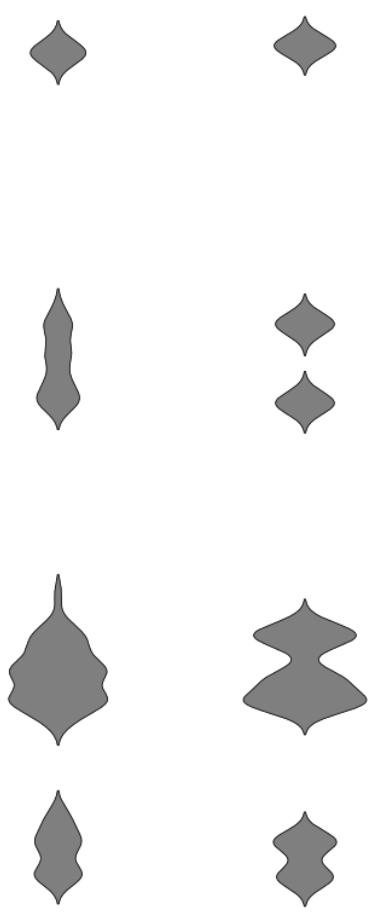

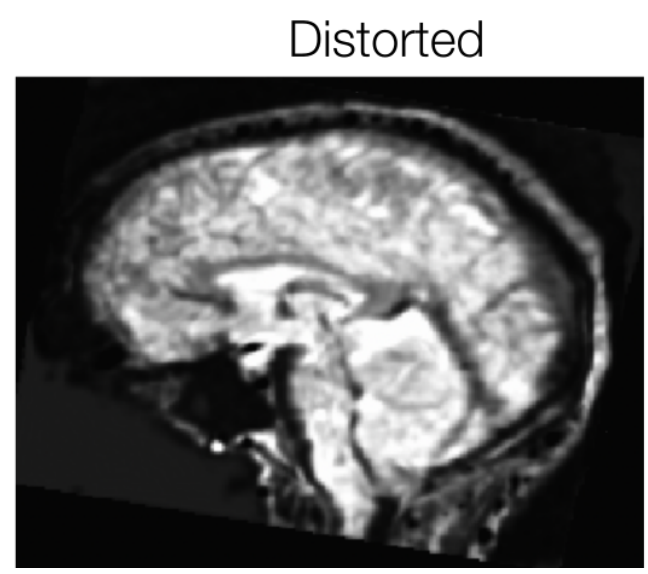

Figure 10: EPI distortion reduces image alignment accuracy. Distributions of $\mathbf{R R}^{-1}$ for $10 \mathrm{MSC}$ subjects. Left column depicts the distribution of affine alignment errors (2-norm of the $\mathbf{R R}^{-1}$ product) when no distortion is present. The right column depicts affine alignment errors for the same images when phase-encoding direction error is present. Below each column: example images depicting the distorted and undistorted images used in this analysis. Notice that in this example the phase encoding distortions are quite subtle yet still produce significant variability in affine alignment performance. 


\section{References}

[1] J. L. Andersson, S. Skare, J. Ashburner, How to correct susceptibility distortions in spin-echo echoplanar images: application to diffusion tensor imaging, NeuroImage 20 (2) (2003) 870-888. doi: $10.1016 / \mathrm{s} 1053-8119(03) 00336-7$.

URL https://dx.doi.org/10.1016/s1053-8119(03)00336-7

[2] J. L. Andersson, S. Skare, A Model-Based Method for Retrospective Correction of Geometric Distortions in Diffusion-Weighted EPI, NeuroImage 16 (1) (2002) 177-199. doi:10.1006/nimg.2001.1039. URL https://dx.doi.org/10.1006/nimg.2001.1039

[3] P. Jezzard, A. S. Barnett, C. Pierpaoli, Characterization of and correction for eddy current artifacts in echo planar diffusion imaging, Magnetic Resonance in Medicine 39 (5) (1998) 801-812. doi:10.1002/ mrm. 1910390518.

URL https://dx.doi.org/10.1002/mrm.1910390518

[4] P. Jezzard, R. S. Balaban, Correction for geometric distortion in echo planar images from B0 field variations, Magnetic Resonance in Medicine 34 (1) (1995) 65-73. doi:10.1002/mrm. 1910340111. URL https://dx.doi.org/10.1002/mrm.1910340111

[5] E. W. Dickie, A. Anticevic, D. E. Smith, T. S. Coalson, M. Manogaran, N. Calarco, J. D. Viviano, M. F. Glasser, D. C. V. Essen, A. N. Voineskos, Ciftify: A framework for surface-based analysis of legacy MR acquisitions, NeuroImage 197 (2019) 818-826. doi:10.1016/j.neuroimage.2019.04.078. URL https://dx.doi.org/10.1016/j.neuroimage.2019.04.078

[6] R. Cusack, M. Brett, K. Osswald, An Evaluation of the Use of Magnetic Field Maps to Undistort Echo-Planar Images, NeuroImage 18 (1) (2003) 127-142. doi:10.1006/nimg.2002.1281.

URL https://dx.doi.org/10.1006/nimg.2002.1281

[7] H. Togo, J. Rokicki, K. Yoshinaga, T. Hisatsune, H. Matsuda, N. Haga, T. Hanakawa, Effects of FieldMap Distortion Correction on Resting State Functional Connectivity MRI, Frontiers in Neuroscience 11 (2017). doi:10.3389/fnins.2017.00656.

URL https://dx.doi.org/10.3389/fnins.2017.00656

[8] J. L. Andersson, S. N. Sotiropoulos, An integrated approach to correction for off-resonance effects and subject movement in diffusion MR imaging, NeuroImage 125 (2016) 1063-1078. doi:10.1016/j. neuroimage.2015.10.019. URL https://dx.doi.org/10.1016/j.neuroimage.2015.10.019

[9] A. Gholipour, N. Kehtarnavaz, K. Gopinath, R. Briggs, Cross-Validation of Deformable Registration With Field Maps in Functional Magnetic Resonance Brain Imaging, IEEE Journal of Selected Topics in Signal Processing 2 (6) (2008) 854-869. doi:10.1109/jstsp.2008.2007824.

URL https://dx.doi.org/10.1109/jstsp.2008.2007824

[10] A. Gholipour, N. Kehtarnavaz, R. W. Briggs, K. S. Gopinath, W. Ringe, A. Whittemore, S. Cheshkov, K. Bakhadirov, Validation of Non-Rigid Registration Between Functional and Anatomical Magnetic Resonance Brain Images, IEEE Transactions on Biomedical Engineering 55 (2) (2008) 563-571. doi: 10.1109/tbme.2007.912641.

URL https://dx.doi.org/10.1109/tbme.2007.912641

[11] J. Kybic, P. Thevenaz, A. Nirkko, M. Unser, Unwarping of unidirectionally distorted EPI images, IEEE Transactions on Medical Imaging 19 (2) (2000) 80-93. doi:10.1109/42.836368.

URL https://dx.doi.org/10.1109/42.836368

[12] C. Studholme, R. T. Constable, J. S. Duncan, Accurate alignment of functional EPI data to anatomical MRI using a physics-based distortion model, IEEE Transactions on Medical Imaging 19 (11) (2000) 1115-1127. doi:10.1109/42.896788. URL https://dx.doi.org/10.1109/42.896788

[13] T. Rohlfing, Image Similarity and Tissue Overlaps as Surrogates for Image Registration Accuracy: Widely Used but Unreliable, IEEE Transactions on Medical Imaging 31 (2) (2012) 153-163. doi: 10.1109/tmi . 2011.2163944.

URL https://dx.doi.org/10.1109/tmi.2011.2163944

[14] M. C. Chambers, C. Bhushan, J. P. Haldar, R. M. Leahy, D. W. Shattuck, Correcting inhomogeneity- 
induced distortion in FMRI using non-rigid registration, 2015 IEEE 12th International Symposium on Biomedical Imaging (ISBI) (2015) 1364-1367.

[15] X. Hong, X. V. To, I. Teh, J. R. Soh, K.-H. Chuang, Evaluation of EPI distortion correction methods for quantitative MRI of the brain at high magnetic field, Magnetic Resonance Imaging 33 (9) (2015) 1098-1105. doi:10.1016/j.mri.2015.06.010. URL https://dx.doi.org/10.1016/j.mri.2015.06.010

[16] J. M. Huntenburg (2014).

[17] S. Wang, D. J. Peterson, J. C. Gatenby, W. Li, T. J. Grabowski, T. M. Madhyastha, Evaluation of Field Map and Nonlinear Registration Methods for Correction of Susceptibility Artifacts in Diffusion MRI, Frontiers in Neuroinformatics 11 (2017). doi:10.3389/fninf .2017.00017. URL https://dx.doi.org/10.3389/fninf.2017.00017

[18] J. B. West, J. Fitzpatrick, M. Y. Wang, B. M. Dawant, C. R. Maurer, Jr, R. M. Kessler, R. J. Maciunas, C. Barillot, D. Lemoine, A. M. F. Collignon, F. Maes, P. Suetens, D. Vandermeulen, P. A. V. D. Elsen, P. F. Hemler, S. Napel, T. S. Sumanaweera, B. A. Harkness, D. L. Hill, C. Studholme, G. Malandain, X. Pennec, M. E. Noz, G. Q. Maguire, Jr, M. Pollack, C. A. Pelizzari, R. A. Robb, D. P. Hanson, R. P. Woods, Comparison and evaluation of retrospective intermodality image registration techniques, Image Processing (1996).

[19] T. L. Jernigan, S. A. Brown, G. J. Dowling, The adolescent brain cognitive development study, Journal of research on adolescence: the official journal of the Society for Research on Adolescence 28 (1) (2018) 154.

[20] S. Marek, B. Tervo-Clemmens, A. N. Nielsen, M. D. Wheelock, R. L. Miller, T. O. Laumann, E. Earl, W. W. Foran, M. Cordova, O. Doyle, A. Perrone, O. Miranda-Dominguez, E. Feczko, D. Sturgeon, A. Graham, R. Hermosillo, K. Snider, A. Galassi, B. J. Nagel, S. W. F. Ewing, A. T. Eggebrecht, H. Garavan, A. M. Dale, D. J. Greene, D. M. Barch, D. A. Fair, B. Luna, N. U. Dosenbach, Identifying reproducible individual differences in childhood functional brain networks: An ABCD study, Developmental Cognitive Neuroscience 40 (2019) 100706-100706. doi:10.1016/j.dcn.2019.100706. URL https://dx.doi.org/10.1016/j.dcn.2019.100706

[21] E. M. Gordon, T. O. Laumann, A. W. Gilmore, D. J. Newbold, D. J. Greene, J. J. Berg, M. Ortega, C. Hoyt-Drazen, C. Gratton, H. Sun, J. M. Hampton, R. S. Coalson, A. L. Nguyen, K. B. Mcdermott, J. S. Shimony, A. Z. Snyder, B. L. Schlaggar, S. E. Petersen, S. M. Nelson, N. U. F. Dosenbach (2017). [link].

URL https://doi.org/10.1016/j.neuron.2017.07.011

[22] AFNI program: 3dQwarp.

[23] B. AVANTS, C. EPSTEIN, M. GROSSMAN, J. GEE, Symmetric diffeomorphic image registration with cross-correlation: Evaluating automated labeling of elderly and neurodegenerative brain, Medical Image Analysis 12 (1) (2008) 26-41. doi:10.1016/j.media.2007.06.004.

URL https://dx.doi.org/10.1016/j.media.2007.06.004

[24] FNIRT/UserGuide - FslWiki.

[25] B. B. Avants, N. J. Tustison, G. Song, P. A. Cook, A. Klein, J. C. Gee, A reproducible evaluation of ANTs similarity metric performance in brain image registration, NeuroImage 54 (3) (2011) 2033-2044. doi:10.1016/j.neuroimage.2010.09.025.

URL https://dx.doi.org/10.1016/j.neuroimage.2010.09.025

[26] A. Klein, J. Andersson, B. A. Ardekani, J. Ashburner, B. Avants, M.-C. Chiang, G. E. Christensen, D. L. Collins, J. Gee, P. Hellier, J. H. Song, M. Jenkinson, C. Lepage, D. Rueckert, P. Thompson, T. Vercauteren, R. P. Woods, J. J. Mann, R. V. Parsey, Evaluation of 14 nonlinear deformation algorithms applied to human brain MRI registration, NeuroImage 46 (3) (2009) 786-802. doi: $10.1016 /$ j.neuroimage.2008.12.037.

URL https://dx.doi.org/10.1016/j.neuroimage.2008.12.037

[27] R. M. Braga, R. L. Buckner, Parallel Interdigitated Distributed Networks within the Individual Estimated by Intrinsic Functional Connectivity, Neuron 95 (2) (2017) 457-471.e5. doi : 10.1016/j .neuron . 2017.06 .038 . 
URL https://dx.doi.org/10.1016/j.neuron.2017.06.038

[28] C. Gratton, T. O. Laumann, A. N. Nielsen, D. J. Greene, E. M. Gordon, A. W. Gilmore, S. M. Nelson, R. S. Coalson, A. Z. Snyder, B. L. Schlaggar, N. U. Dosenbach, S. E. Petersen, Functional Brain Networks Are Dominated by Stable Group and Individual Factors, Not Cognitive or Daily Variation, Neuron 98 (2) (2018) 439-452.e5. doi:10.1016/j.neuron.2018.03.035. URL https://dx.doi.org/10.1016/j.neuron.2018.03.035

[29] T. O. Laumann, E. M. Gordon, B. Adeyemo, A. Z. Snyder, S. J. Joo, M.-Y. Chen, A. W. Gilmore, K. B. McDermott, S. M. Nelson, N. U. F. Dosenbach, B. L. Schlaggar, J. A. Mumford, R. A. Poldrack, S. E. Petersen, Functional System and Areal Organization of a Highly Sampled Individual Human Brain, Neuron 87 (3) (2015) 657-670. doi:10.1016/j.neuron.2015.06.037. URL https://dx.doi.org/10.1016/j.neuron.2015.06.037

[30] C. J. Lynch, J. D. Power, M. A. Scult, M. Dubin, F. M. Gunning, C. Liston, Rapid Precision Functional Mapping of Individuals Using Multi-Echo fMRI, Cell Reports 33 (12) (2020) 108540-108540. doi: 10.1016/j.celrep.2020.108540.

URL https://dx.doi.org/10.1016/j.celrep.2020.108540

[31] S. Marek, J. S. Siegel, E. M. Gordon, R. V. Raut, C. Gratton, D. J. Newbold, M. Ortega, T. O. Laumann, B. Adeyemo, D. B. Miller, A. Zheng, K. C. Lopez, J. J. Berg, R. S. Coalson, A. L. Nguyen, D. Dierker, A. N. Van, C. R. Hoyt, K. B. McDermott, S. A. Norris, J. S. Shimony, A. Z. Snyder, S. M. Nelson, D. M. Barch, B. L. Schlaggar, M. E. Raichle, S. E. Petersen, D. J. Greene, N. U. Dosenbach, Spatial and Temporal Organization of the Individual Human Cerebellum, Neuron 100 (4) (2018) 977993.e7. doi:10.1016/j.neuron.2018.10.010.

URL https://dx.doi.org/10.1016/j.neuron.2018.10.010

[32] D. J. Newbold, T. O. Laumann, C. R. Hoyt, J. M. Hampton, D. F. Montez, R. V. Raut, M. Ortega, A. Mitra, A. N. Nielsen, D. B. Miller, B. Adeyemo, A. L. Nguyen, K. M. Scheidter, A. B. Tanenbaum, A. N. Van, S. Marek, B. L. Schlaggar, A. R. Carter, D. J. Greene, E. M. Gordon, M. E. Raichle, S. E. Petersen, A. Z. Snyder, N. U. Dosenbach, Plasticity and Spontaneous Activity Pulses in Disused Human Brain Circuits, Neuron 107 (3) (2020) 580-589.e6. doi:10.1016/j.neuron.2020.05.007. URL https://dx.doi.org/10.1016/j.neuron.2020.05.007

[33] C. M. Sylvester, Q. Yu, A. B. Srivastava, S. Marek, A. Zheng, D. Alexopoulos, C. D. Smyser, J. S. Shimony, M. Ortega, D. L. Dierker, G. H. Patel, S. M. Nelson, A. W. Gilmore, K. B. McDermott, J. J. Berg, A. T. Drysdale, M. T. Perino, A. Z. Snyder, R. V. Raut, T. O. Laumann, E. M. Gordon, D. M. Barch, C. E. Rogers, D. J. Greene, M. E. Raichle, N. U. F. Dosenbach, Individual-specific functional connectivity of the amygdala: A substrate for precision psychiatry, Proceedings of the National Academy of Sciences 117 (7) (2020) 3808-3818. doi:10.1073/pnas. 1910842117. URL https://dx.doi.org/10.1073/pnas.1910842117

[34] A. Zheng, D. F. Montez, S. Marek, A. W. Gilmore, D. J. Newbold, T. O. Laumann, B. P. Kay, N. A. Seider, A. N. Van, J. M. Hampton, D. Alexopolous, B. L. Schlaggar, C. M. Sylvester, D. J. Greene, J. S. Shimony, S. M. Nelson, G. S. Wig, C. Gratton, K. B. Mcdermott, M. E. Raichle, E. M. Gordon, N. U. F. Dosenbach (2020). [link]. URL https://doi.org/10.1101/2020.12.01.395210

[35] B. J. Casey, T. Cannonier, M. I. Conley, A. O. Cohen, D. M. Barch, M. M. Heitzeg, M. E. Soules, T. Teslovich, D. V. Dellarco, H. Garavan, C. A. Orr, T. D. Wager, M. T. Banich, N. K. Speer, M. T. Sutherland, M. C. Riedel, A. S. Dick, J. M. Bjork, K. M. Thomas, B. Chaarani, M. H. Mejia, D. J. Hagler, M. D. Cornejo, C. S. Sicat, M. P. Harms, N. U. Dosenbach, M. Rosenberg, E. Earl, H. Bartsch, R. Watts, J. R. Polimeni, J. M. Kuperman, D. A. Fair, A. M. Dale, The Adolescent Brain Cognitive Development (ABCD) study: Imaging acquisition across 21 sites, Developmental Cognitive Neuroscience 32 (2018) 43-54. doi:10.1016/j.dcn.2018.03.001. URL https://dx.doi.org/10.1016/j.dcn.2018.03.001

[36] FLIRT/UserGuide - FslWiki.

[37] D. N. Greve, B. Fischl, Accurate and robust brain image alignment using boundary-based registration, NeuroImage 48 (1) (2009) 63-72. doi:10.1016/j.neuroimage.2009.06.060. 
URL https://dx.doi.org/10.1016/j.neuroimage.2009.06.060

[38] J. M. Treiber, N. S. White, T. C. Steed, H. Bartsch, D. Holland, N. Farid, C. R. McDonald, B. S. Carter, A. M. Dale, C. C. Chen, Characterization and Correction of Geometric Distortions in 814 Diffusion Weighted Images, PLOS ONE 11 (3) (2016) e0152472-e0152472. doi:10.1371/journal. pone. 0152472 .

URL https://dx.doi.org/10.1371/journal.pone.0152472

[39] R. W. Cox, AFNI: software for analysis and visualization of functional magnetic resonance neuroimages, Comput. Biomed. Res 29 (1996) 162-173.

[40] J. T. Devlin, R. P. Russell, M. H. Davis, C. J. Price, J. Wilson, H. E. Moss, P. M. Matthews, L. K. Tyler, Susceptibility-Induced Loss of Signal: Comparing PET and fMRI on a Semantic Task, NeuroImage 11 (6) (2000) 589-600. doi:10.1006/nimg.2000.0595.

URL https://dx.doi.org/10.1006/nimg.2000.0595

[41] M. Visser, K. V. Embleton, E. Jefferies, G. J. Parker, M. L. Ralph, The inferior, anterior temporal lobes and semantic memory clarified: Novel evidence from distortion-corrected fMRI, Neuropsychologia 48 (6) (2010) 1689-1696. doi:10.1016/j.neuropsychologia.2010.02.016.

URL https://dx.doi.org/10.1016/j.neuropsychologia.2010.02.016

[42] A. Sotiras, C. Davatzikos, N. Paragios, Deformable Medical Image Registration: A Survey, IEEE Transactions on Medical Imaging 32 (7) (2013) 1153-1190. doi:10.1109/tmi.2013.2265603. URL https://dx.doi.org/10.1109/tmi.2013.2265603

[43] P. Jezzard, S. Clare, Sources of distortion in functional MRI data, Human Brain Mapping 8 (2-3) (1999) 80-85. doi:10.1002/(sici) 1097-0193(1999) 8:2/3<80: :aid-hbm2>3.0.co;2-c. URL https://dx.doi.org/10.1002/(sici)1097-0193(1999)8:2/3<80::aid-hbm2>3.0.co;2-c

[44] X. Zhang, J. Jiang, S. Peng, Commutability of blur and affine warping in super-resolution with application to joint estimation of triple-coupled variables, IEEE Trans. Image Process 21 (2012) 1796-1808.

[45] S. Marek, B. Tervo-Clemmens, F. J. Calabro, D. F. Montez, B. P. Kay, A. S. Hatoum, M. R. Donohue, W. Foran, R. L. Miller, E. Feczko, O. Miranda-Dominguez, A. M. Graham, E. A. Earl, A. J. Perrone, M. Cordova, O. Doyle, L. A. Moore, G. Conan, J. Uriarte, K. Snider, A. Tam, J. Chen, D. J. Newbold, A. Zheng, N. A. Seider, A. N. Van, T. O. Laumann, W. K. Thompson, D. J. Greene, S. E. Petersen, T. E. Nichols, B. T. T. Yeo, D. M. Barch, H. Garavan, B. Luna, D. A. Fair, N. U. F. Dosenbach (2020). [link].

URL https://doi.org/10.1101/2020.08.21.257758

[46] N. J. Tustison, B. B. Avants, P. A. Cook, Y. Zheng, A. Egan, P. A. Yushkevich, J. C. Gee, N4ITK: Improved N3 Bias Correction, IEEE Transactions on Medical Imaging 29 (6) (2010) 1310-1320. doi: 10.1109/tmi.2010.2046908.

URL https://dx.doi.org/10.1109/tmi.2010.2046908

[47] S. M. Smith, Fast robust automated brain extraction, Hum. Brain Mapp 17 (2002) 143-155.

[48] B. Fischl, FreeSurfer, NeuroImage 62 (2) (2012) 774-781. doi:10.1016/j.neuroimage.2012.01.021. URL https://dx.doi.org/10.1016/j.neuroimage.2012.01.021

[49] C. D. Smyser, T. E. Inder, J. S. Shimony, J. E. Hill, A. J. Degnan, A. Z. Snyder, J. J. Neil, Longitudinal analysis of neural network development in preterm infants, Cerebral cortex 20 (12) (2010) 2852-2862.

[50] J. D. Power, K. A. Barnes, A. Z. Snyder, B. L. Schlaggar, S. E. Petersen, Spurious but systematic correlations in functional connectivity MRI networks arise from subject motion, NeuroImage 59 (3) (2012) 2142-2154. doi:10.1016/j.neuroimage.2011.10.018. URL https://dx.doi.org/10.1016/j.neuroimage.2011.10.018

[51] Y. Behzadi, K. Restom, J. Liau, T. T. Liu, A component based noise correction method (CompCor) for BOLD and perfusion based fMRI, NeuroImage 37 (1) (2007) 90-101. doi:10.1016/j.neuroimage. 2007.04 .042$.

URL https://dx.doi.org/10.1016/j.neuroimage.2007.04.042

[52] N. V. Petersen, J. L. Jensen, J. Burchhardt, H. Stødkilde-Jørgensen, State Space Models for Physiological Noise in fMRI Time Series, NeuroImage 7 (4) (1998) S592-S592. doi:10.1016/s1053-8119(18) 31425-3. 
URL https://dx.doi.org/10.1016/s1053-8119(18)31425-3

[53] L. S. King, M. C. Camacho, D. F. Montez, K. L. Humphreys, I. H. Gotlib, Naturalistic language input is associated with resting-state functional connectivity in infancy, Journal of Neuroscience 41 (3) (2021) 424-434.

[54] R. V. Raut, A. Mitra, S. Marek, M. Ortega, A. Z. Snyder, A. Tanenbaum, T. O. Laumann, N. U. Dosenbach, M. E. Raichle, Organization of propagated intrinsic brain activity in individual humans, Cerebral Cortex 30 (3) (2020) 1716-1734.

[55] N. Otsu, A Threshold Selection Method from Gray-Level Histograms, IEEE Transactions on Systems, Man, and Cybernetics 9 (1) (1979) 62-66. doi:10.1109/tsmc.1979.4310076.

URL https://dx.doi.org/10.1109/tsmc.1979.4310076

[56] B. Lipschutz, K. J. Friston, J. Ashburner, R. Turner, C. J. Price, Assessing Study-Specific Regional Variations in fMRI Signal, NeuroImage 13 (2) (2001) 392-398. doi:10.1006/nimg.2000.0687. URL https://dx.doi.org/10.1006/nimg.2000.0687

[57] P. Jain, P. Kar (2017). [link]. URL https://doi.org/10.1561/9781680833690

[58] J. Chung, E. Haber, J. Nagy (2006). [link]. URL https://doi.org/10.1088/0266-5611/22/4/009

[59] T. Dozat, Incorporating Nesterov Momentum into Adam. (2016). 